\title{
The two-pore domain potassium channel TREK-1 mediates cardiac fibrosis and diastolic dysfunction
}

\author{
Dennis M. Abraham, ${ }^{1}$ Teresa E. Lee, ${ }^{2}$ Lewis J. Watson, ${ }^{1}$ Lan Mao, ${ }^{1}$ Gurangad Chandok, ${ }^{1}$ Hong-Cang Wang, ${ }^{1}$ Stephan Frangakis, ${ }^{2}$ \\ Geoffrey S. Pitt, ${ }^{1}$ Svati H. Shah, ${ }^{1}$ Matthew J. Wolf, ${ }^{1}$ and Howard A. Rockman ${ }^{1,2,3}$
}

'Department of Medicine, 2Department of Cell Biology, and ${ }^{2}$ Department of Molecular Cenetics and Microbiology, Duke University Medical Center, Durham, North Carolina, USA

\begin{abstract}
Cardiac two-pore domain potassium channels (K2P) exist in organisms from Drosophila to humans; however, their role in cardiac function is not known. We identified a K2P gene, CG8713 (sandman), in a Drosophila genetic screen and show that sandman is critical to cardiac function. Mice lacking an ortholog of sandman, TWIK-related potassium channel (TREK-1, also known Kcnk2), exhibit exaggerated pressure overload-induced concentric hypertrophy and alterations in fetal gene expression, yet retain preserved systolic and diastolic cardiac function. While cardiomyocyte-specific deletion of TREK- 1 in response to in vivo pressure overload resulted in cardiac dysfunction, TREK-1 deletion in fibroblasts prevented deterioration in cardiac function. The absence of pressure overload-induced dysfunction in TREK-1-KO mice was associated with diminished cardiac fibrosis and reduced activation of JNK in cardiomyocytes and fibroblasts. These findings indicate a central role for cardiac fibroblast TREK- 1 in the pathogenesis of pressure overload-induced cardiac dysfunction and serve as a conceptual basis for its inhibition as a potential therapy.
\end{abstract}

\section{Introduction}

Two-pore domain potassium channels (K2P) are 4-transmembrane-spanning and two-pore-forming domain proteins (1) that contribute to resting membrane potential in excitable tissue (2). Two K2P subfamily members, TWIK-related acid-sensitive potassium channel (TASK) and TWIK-related potassium channel (TREK) channels, are highly expressed in human cardiac tissue (3) and implicated in cardiac arrhythmogenesis (4). TREK channels, which are present in atrial (5) and ventricular tissue $(6,7)$, have been of marked interest because of their role in cardiac chronotropic function (8), dynamic modulation by adrenergic signaling (9), and biomechanical stretch (10). Recent work has identified K2P channels as significant modulators of Drosophila diastolic function (11). However, the impact of TREK channels on mammalian ventricular systolic and diastolic function is unknown.

Cardiac hypertrophy and concomitant cardiac fibrosis are frequently associated with the development of congestive heart failure, particularly heart failure with preserved ejection fraction (HFpEF), which is associated with significant mortality $(12,13)$. HFpEF patients frequently exhibit left ventricular (LV) geometry, characterized by normal end-diastolic volume and concentric cardiac hypertrophy $(14,15)$. This structural phenotype is closely associated with the development of diastolic dysfunction $(16,17)$, manifested as abnormalities in both active myocardial relaxation (18) and ventricular distensibility (19-21). The latter is thought to arise from a combination of myocyte stiffness and increased myocardial fibrosis (22).

Conflict of interest: The authors have declared that no conflict of interest exists. License: Copyright 2018, American Society for Clinical Investigation.

Submitted: June 26, 2017; Accepted: August 23, 2018.

Reference information: J Clin Invest. 2018;128(11):4843-4855.

https://doi.org/10.1172/JCI95945
The development of cardiac fibrosis has long been considered a pathological process associated with cardiac hypertrophy and diastolic dysfunction (23). While cardiac fibrosis develops in response to tissue injury and is necessary for healing (24), excessive fibrosis can disrupt normal cardiac architecture and result in enhanced arrhythmogenesis (25), impaired vasomotor function (26), and abnormal ventricular function (27). In turn, these pathologic changes form the substrate for increased sudden cardiac death and progressive heart failure mortality $(28,29)$. There is a growing literature describing biochemical crosstalk between cardiac myocytes and fibroblasts that influence both cardiac morphology and function (30). K2P channels have been implicated in diastolic function in Drosophila; however, the contribution of $\mathrm{K} 2 \mathrm{P}$ channels in mammalian cardiac function is unknown. Here, we show that the K2P channel TREK-1 (also known Kcnk2) is a key modulator of both cardiac hypertrophy and diastolic function through distinct effects on both cardiac myocytes and fibroblasts.

\section{Results}

Drosophila genetic screen identifies sandman as a cardiomyopathy-causing gene. We performed a genetic screen to identify genes that alter cardiac function in adult Drosophila and identified an enlarged cardiac chamber size and reduced fractional shortening (FS) corresponding to $D f(2 R)$ Exel7094, a heterozygous genomic deficiency of chromosome 2R (Figure 1A). Drosophila harboring the overlapping genomic deficiencies $D f(2 R)$ Exel6055 and $D f(2 R)$ Exel7095 had normal cardiac chamber dimensions, thereby refining the candidate region to approximately $60 \mathrm{~kb}$, which encoded 13 genes (Figure 1A). A mutant with a P-element that disrupted sandman, PBacsandman00867, phenocopied Df(2R)Exel7094 (Figure 1, $\mathrm{B}-\mathrm{E})$. Additionally, the ubiquitous or cardiac-specific expression of a cDNA encoding sandman using an actin-Gal4 or tinC-Gal4 driver, respectively, prevented the cardiac phenotype observed with $D f(2 R)$ 
A

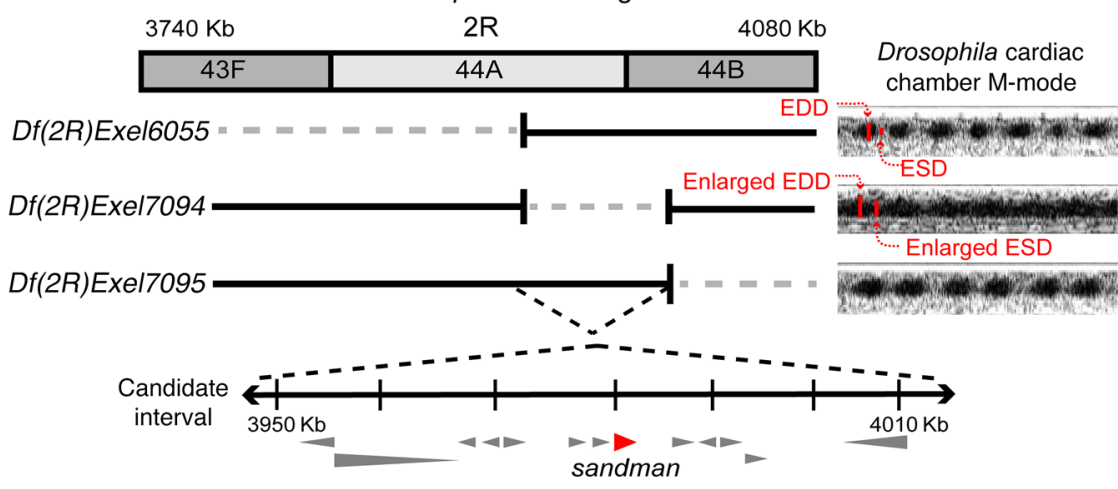

B

Representative Drosophila optical coherence tomography images

w1118

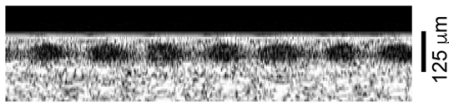

Df(2R)Exel7094 (deletion)

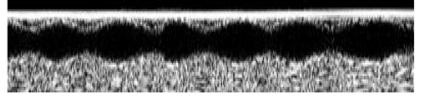

PBac\{RB\}sandman e00867 (p-element)

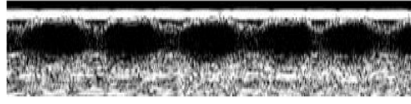

actin>sandman (ubiquitous expression)

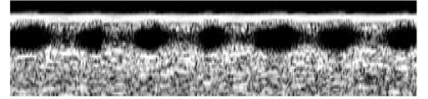

tinC>sandman (cardiac expression)

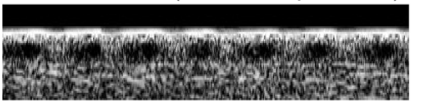

Df(2R)Exel7094; actin>sandman

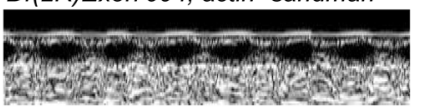

Df(2R)Exel7094; tinC>sandman

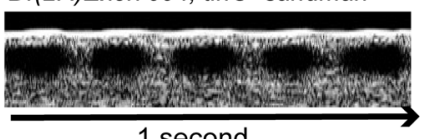

1 second
C

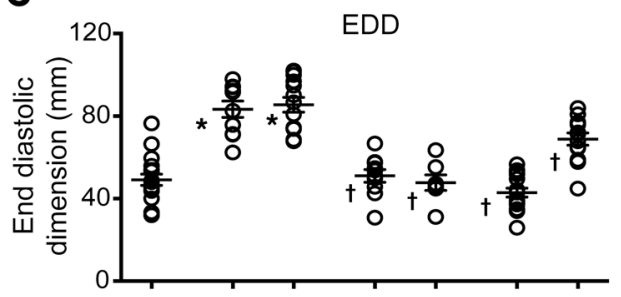

D

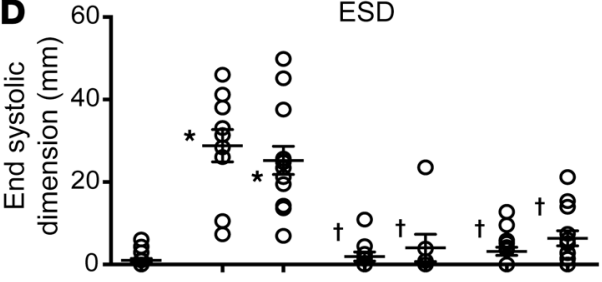

$\mathbf{E}$

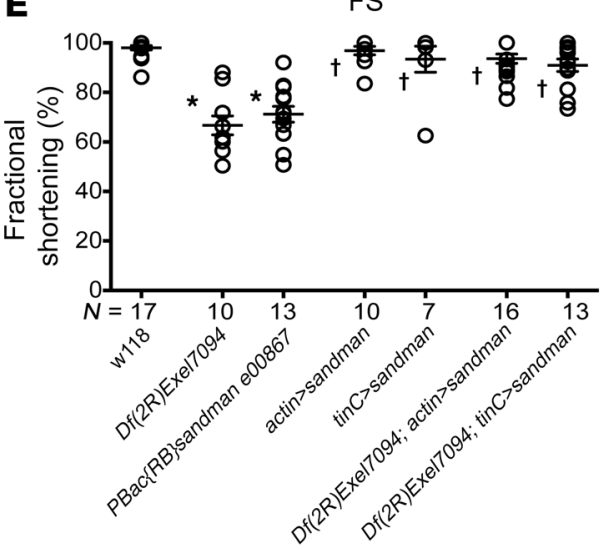

Figure 1. Sandman is critical for Drosophila cardiac function. (A) Schematic of Drosophila genetic screen. Genetic deletion of a segment between cytologic bands 44A and 44B of chromosome 2R, $D f(2 R)$ Exel7094, resulted in enlarged cardiac dimensions and diminished cardiac function by OCT. Adjacent genomic deletions $D f(2 R)$ Exel6055 and $D f(2 R)$ Exel7095 have normal cardiac dimensions, thereby identifying a genomic segment spanning 13 genes as the candidate interval. (B) Representative 1 second OCT recordings from $w 1118$, genomic deletion ( $D f[2 R]$ Exel7094), P-element single-gene disruption of sandman (PBac $\{R B\}$ sandman e00867), sandman overexpressed ubiquitously (actin $>$ sandman), sandman overexpressed in cardiac tissue ( $\operatorname{tin} C>$ sandman) or ubiquitously (actin $>$ sandman), or in the context of the genomic deletion $D f(2 R)$ Exel7094 ( $D f(2 R)$ Exel7094; actin>sandman and $D f(2 R)$ Exel7094; tinC $>$ sandman). Scale bar: $125 \mu \mathrm{m}$. (C) Average end diastolic dimensions (EDD) and (D) average end systolic dimensions (ESD) reveal marked enlargement and resultant decrease in (E) average fractional shortening (FS) in $D f(2 R)$ Exel7094 and $P B a c\{R B\}$ sandman e00867 in comparison with w1118. The ubiquitous and cardiac-specific overexpression of sandman in the context of $D f(2 R)$ Exel7094 ( $D f(2 R)$ Exel7094; actin>sandman and $D f(2 R)$ Exel7094; tinC>sandman, respectively) results in rescue of cardiac dimensions in comparison with $D f(2 R)$ Exel7094 and $P B a c\{R B\}$ sandman e00867. Statistical comparisons made using 1-way ANOVA with Bonferroni's test for multiple comparisons. ${ }^{*} P<$ 0.0001 versus $w 1118 ;{ }^{\dagger} P<0.0001$ versus $D f(2 R)$ Exel7094 and PBac\{RB\}sandman e00867.
Exel7094 (Figure 1, B-E). These findings are supported by recent work showing that sandman, so named due to effects on Drosophila circadian rhythm (31), alters Drosophila cardiac function (11). sandman bears sequence similarity to K2P genes (Supplemental Figure 1; supplemental material available online with this article; https://doi. org/10.1172/JCI95945DS1); therefore, we investigated a mammalian ortholog, TREK-1, in the context of mammalian cardiac function.

Loss of function of TREK-1 shows exacerbated cardiac hypertrophy without systolic dysfunction after pressure overload. Global TREK-1KO mice had reduced cardiac end-diastolic volumes in a basal state, but overall morphology was similar to that of controls (Supplemental Table 1 and Supplemental Table 2). Since TREK-1 is activated by biomechanical stretch (10) and TREK-1 gene expression increases in normal hearts and in cardiac fibroblasts with pressure overload (Supplemental Figure 2A and Supplemental Figure 3), we examined the contribution of TREK-1 to cardiac responses to chronic pressure overload using transverse aortic constriction (TAC) (32). In response to TAC, global TREK-1-KO mice developed enhanced concentric hypertrophy (Figure 2, A, B, E, and F, and Supplemental Table 2), yet cardiac function remained preserved compared with that of WT mice (Figure 2, C and D). Cardiac function in global TREK-1-KO mice remained protected for up to 1 year following TAC (Supplemental Figure 4). The preservation of cardiac function in global TREK-1KO hearts occurred despite induction of fetal genes (Figure 3, A and $\mathrm{B})$, decreased expression of calcium-handling genes (Supplemental Figure 2, B and C), activation of calcineurin, and enhanced $\mathrm{Ca}^{2+}$ / 
A

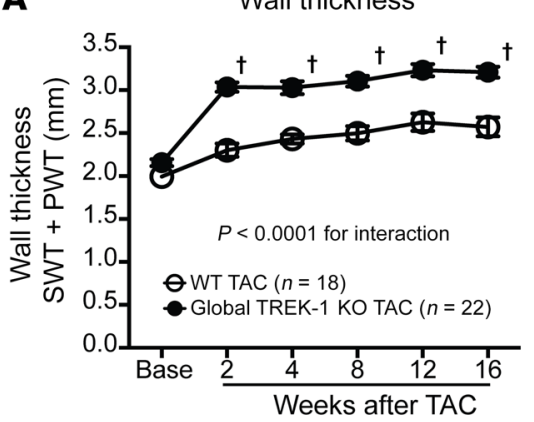

C

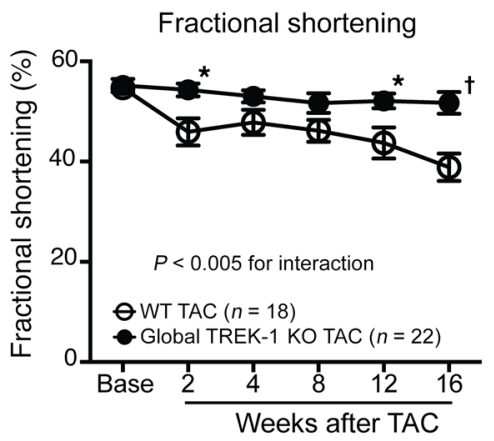

$\mathbf{E}$
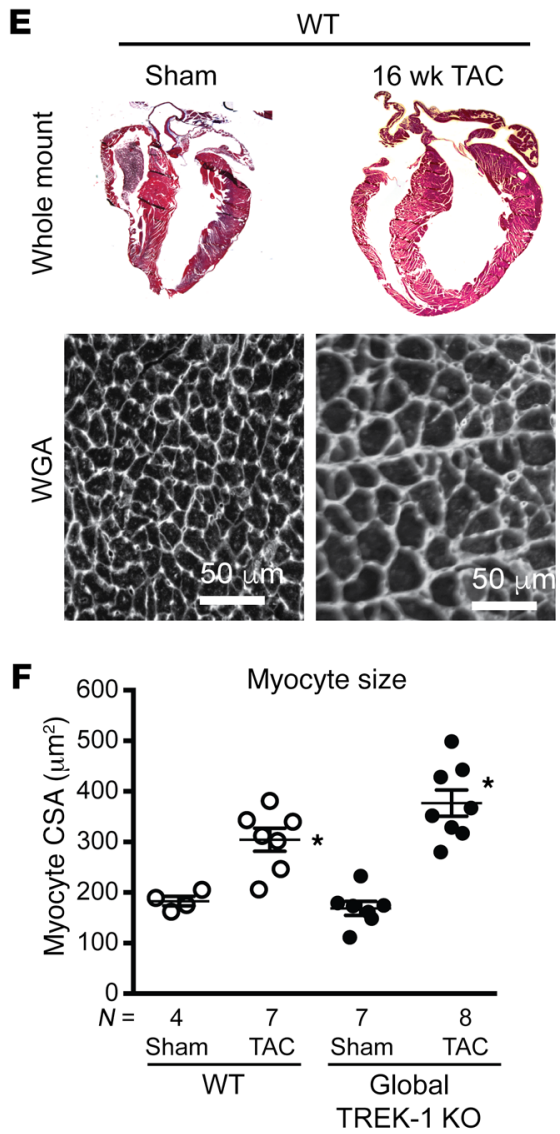

B Change in wall thickness

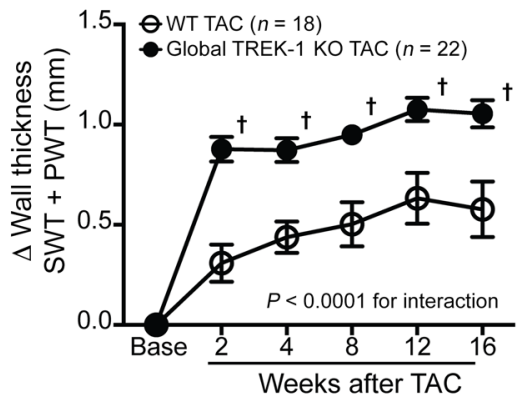

D

Change in fractional shortening
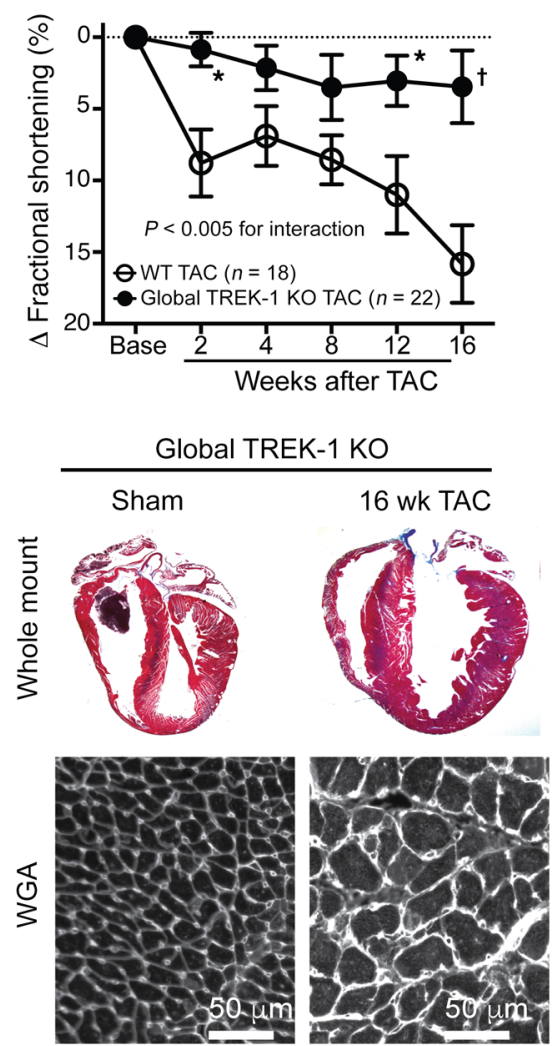

Figure 2. Global TREK-1 KO develops hypertrophy and maintains function after pressure overload.

(A) Serial echocardiographic measurements of average wall thicknesses (SWT + PWT) and (B) average change in wall thickness, (C) average FS ([EDD $-E S D / E D D] \times 100)$ and (D) average change in FS in TREK-1 KO and WT at baseline and up to 16 weeks after TAC. Error bars reflect SEM. Statistical comparisons between WT TAC and TREK-1-KO TAC data were made using 2-way repeated measures ANOVA. $P$ values for the interaction between genotype and weeks after TAC are shown. Comparisons between genotypes at each time point were made using Bonferroni's test for multiple comparisons. ${ }^{\dagger} P<0.001 ;{ }^{*} P<0.05$ for WT TAC versus TREK-1-KO TAC at each time point. (E) Whole mount of hearts from WT and TREK-1-KO hearts both under sham and 16 weeks of TAC conditions. Representative wheat germ agglutinin (WCA) staining showing myocyte areas in WT and TREK-1 KO 16 weeks following sham or TAC. Scale bars: $50 \mu \mathrm{m}$. (F) Average myocyte cross-sectional area (CSA) in WT and TREK-1-KO cross-sectional area after 16 weeks of TAC. The average measured CSAs of $300-500$ cardiomyocytes were used for each animal. Statistical comparisons were performed using 1-way ANOVA with Newman-Keuls test for multiple comparisons. ${ }^{*} P<0.05$ versus WT sham. calmodulin kinase II (CaMKII) activity, as assessed by phospholamban phosphorylation (Figure 3, C and D). Since TREK-1 is a $\mathrm{K}^{+}$ channel that can influence resting membrane potential, we recorded $\mathrm{K}^{+}$currents in isolated cardiomyocytes. We found both reduced current and prolonged action potential duration in TREK-1-
$\mathrm{KO}$ cardiomyocytes, which is consistent with $\mathrm{KO}$ of potassium channel function (Supplemental Figure 5).

Hemodynamic measurements using pressure-volume loop analyses demonstrated that the markedly hypertrophied hearts from global TREK-1-KO mice had enhanced cardiac contractility, 
A

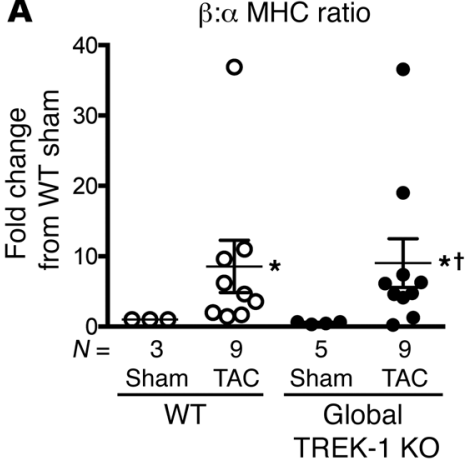

C

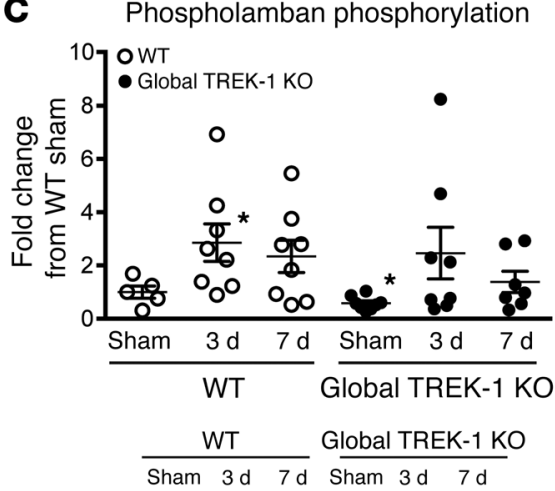

p-Plb (T17)

$\mathrm{t}-\mathrm{Plb}$

GAPDH
B

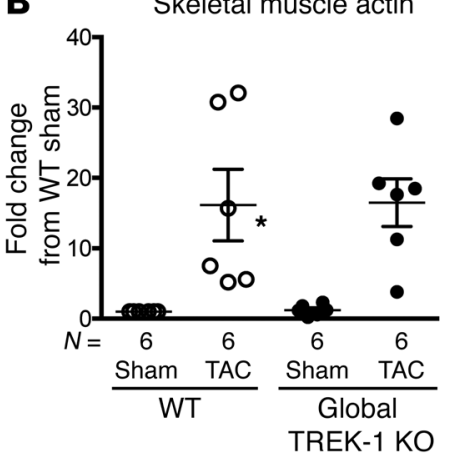

D Calcineurin activity:

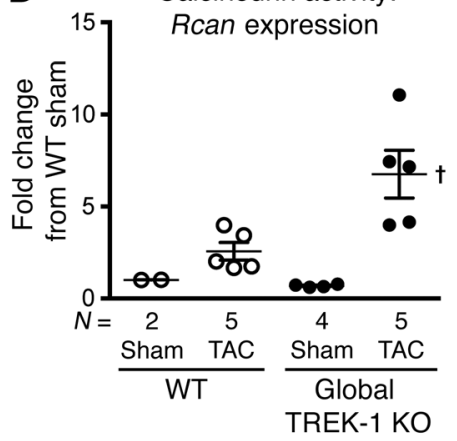

Figure 3. Global TREK-1 KO develops hypertrophic molecular signatures after pressure overload. (A) Average $\beta-M H C$ to $\alpha-M H C$ ratio and (B) skeletal muscle actin gene expression in LVs after 2 weeks of TAC. For gene expression studies in panels $\mathbf{A}$ and $\mathbf{B}$, statistical comparisons with WT sham, which has a theoretical mean of 1 , were made with a 1 -sample, 2 -tailed $t$ test. ${ }^{*} P<0.05$ versus WT sham. Comparisons among all other groups excluding WT sham were made using by Kruskal-Wallis test with Dunn's multiple comparisons test. ${ }^{\dagger} P<0.05$ versus TREK- 1 KO sham. (C) CaMKII activity, as measured by phosphorylated phospholamban at threonine 17. Densitometry quantification of the ratio of phosphorylated phospholamban to total phospholamban, normalized to WT sham condition. Full, uncut gels are shown in the supplemental material. (D) Calcineurin activity as measured by RCAN1 gene expression from cDNA obtained from WT and TREK-1-KO LVs after sham or TAC. For the phospholamban (shown in $\mathbf{C}$ ) and calcineurin experiments (shown D), statistical comparisons with WT sham, which has a theoretical mean of 1 , were made with a 1-sample, 2 -tailed $t$ test. ${ }^{*} P<0.05$ versus WT sham. Comparisons among all other groups excluding WT sham were made using by Kruskal-Wallis test with Dunn's multiple comparisons test. ${ }^{\dagger} P<0.05$ versus WT TAC. with increased load-independent measures of end-systolic pressure-volume relationship (ESPVR) and maximal elastance compared with that of WT mice after 2 weeks of TAC (Figure 4, A, B, and F, and Supplemental Table 3). Consistent with the noninvasive echocardiographic measures of improved cardiac function, TREK-1KO mice showed a $39 \%$ increase in stroke work and preserved cardiac output after TAC (Supplemental Table 1). In addition to enhanced cardiac contractility, TREK-1-KO mice exhibited a preservation of diastolic function after TAC, as measured by active relaxation with a $32 \%$ reduction in the minimum rate of $\mathrm{LV}$ pressure change $(\mathrm{dP} /$ $\left.\mathrm{dt}_{\text {min }}\right)$, and cardiac compliance, as measured by a preserved enddiastolic pressure-volume relationship (EDPVR) (Figure 4, A-E, and Supplemental Table 3). Thus, in response to pathological pressure overload stress, TREK-1-KO mice develop increased concentric hypertrophy and changes in fetal gene expression, but show preserved systolic and diastolic cardiac function.

TREK-1-KO mice show diminished cardiac fibrosis and fibroblast dysfunction. Since cardiac fibroblasts are abundant noncardiomyocyte cells that are known to influence cardiac function (33), we next assessed whether fibroblast function or cardiac fibrosis was altered in global TREK-1-KO mice. In global TREK-1-KO mice, cardiac compliance, which is significantly influenced by cardiac fibrosis, was preserved despite enhanced cardiac hypertrophy (Figure 4, $\mathrm{C}-\mathrm{E}$ ). Both cardiac fibrosis (Figure 5, A and B) and fibrosis gene expression (Figure 5, C and D) were attenuated in global TREK-1KO mice after 2 weeks of TAC. We tested to determine whether the lack of fibrotic response was impaired in noncardiac tissue using both an in vivo and an in vitro wound-healing model. Both in vitro scratch closure of fibroblasts isolated from global TREK-1-KO lungs (Figure $5 \mathrm{E}$ ) and in vivo cutaneous wound healing were significantly diminished in global TREK-1 mice (Figure $5 \mathrm{~F}$ ), indicating a reduction in fibroblast proliferation and migration. There was no significant difference between TREK-1-KO and WT fibroblast transdifferentiation to myofibroblasts (Supplemental Figure 6). These data identify significant impairments in the development of both cardiac and noncardiac fibrosis in TREK-1-KO mice.

Fibroblast-specific loss of TREK-1 protects against pressure overload-induced cardiac dysfunction. To determine whether the protective effect of global TREK-1 loss of function is mediated by cardiomyocytes or fibroblasts, we generated cardiomyocyte-specific and fibroblast-specific deletions of TREK-1 and examined the effect of TAC-induced pressure overload. Conditional TREK-1$\mathrm{KO}$ mice were generated using a targeting strategy that deleted the gene segment between exons 2 and 3 (Figure 6A). Mice bearing the conditional KO allele $\left(K c n k 2^{f / f f}\right)$ were crossed with mice bearing a cre recombinase under the control of either $\alpha$-myosin heavy chain ( $a M H C-c r e)$ or tcf21-iCre, resulting in cardiomyocyte- $\left(\right.$ aMHC-cre;Kcnk2 $\left.2^{t / f f}\right)$ or fibroblast-specific loss of TREK-1 $\left(t c f 21-i C r e ; K c n k 2^{f / f l}\right)$. In sharp contrast to either Kcnk2 $2^{f / f l}$ only or aMHC-cre;Kcnk $2^{f / f l}$ mice, $t c f 21-i C r e ; K c n k 2^{f / f f l}$ mice had preservation of systolic function (Figure 6, B and C, and Supplemental Table 4) and ventricular dimensions (Figure 6D and Supplemental Table 4) after TAC. The preservation of cardiac function and chamber size in $t c f 21-i C r e ; K c n k 2^{f / f l}$ mice was associated with significantly less 
A

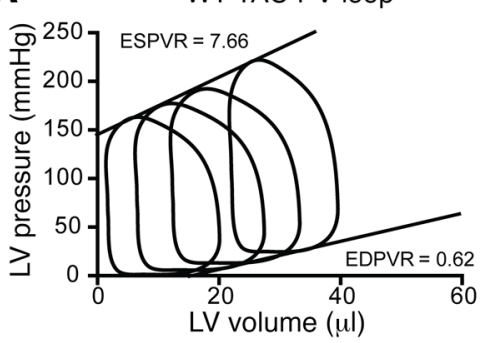

C WT cardiac compliance

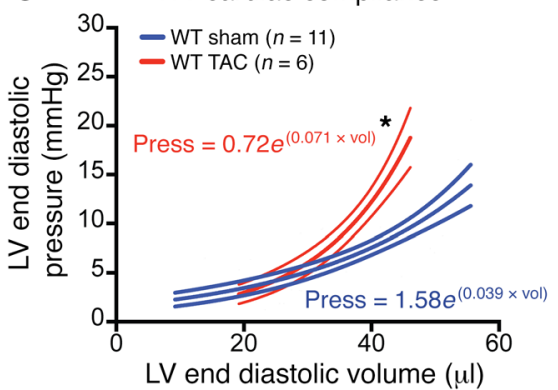

E End diastolic PV relationship

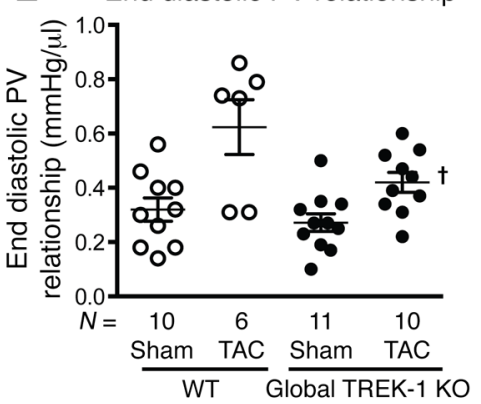

B Global TREK-1 KO TAC PV loop

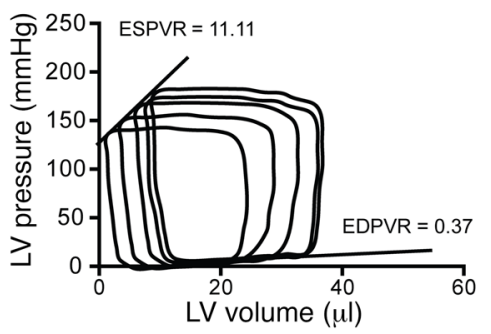

D Global TREK-1 KO cardiac compliance

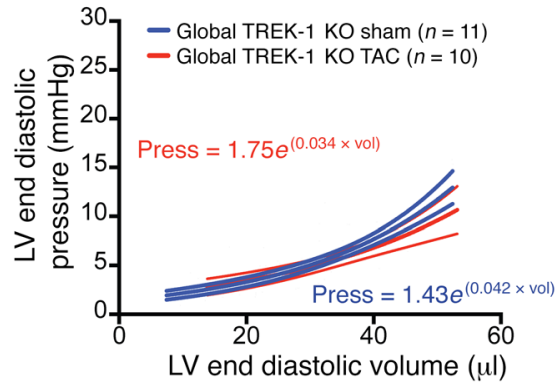

$\mathbf{F}$

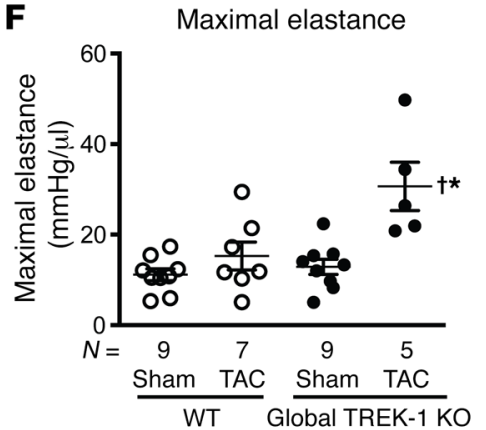

Figure 4. TREK-1 modulates cardiac function. Representative pressure-volume loops obtained from (A) WT mice and (B) TREK-1 KO mice after 2 weeks of TAC. Average slopes of ESPVR and EDPVR are listed for each genotype. (C) Passive diastolic stiffness derived from the following exponential equation: (LV end diastolic pressure $=$ curve fitting constant $\times$

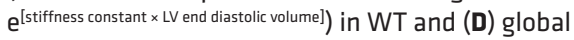
TREK-1-KO mice in sham and TAC states. ${ }^{*} P<0.0001$ versus sham condition curve. Error bars represent $95 \% \mathrm{Cl}$. Pressure $($ Press) $=$ curve fitting constant $x$ e(stiffness constant $\times$ LV end diastolic volume [Vol]). (E) Average end diastolic PV relation and (F) average maximal elastance in sham and 2 weeks of TAC. ${ }^{*} P<0.05$ versus WT sham using 1 -sample, 2 -tailed $t$ test; ${ }^{\dagger} P<0.05$ versus WT TAC by Kruskal-Wallis test with Dunn's multiple comparisons test. interstitial fibrosis when compared with that in pressure-loaded $K c n k 2^{f l / f l}$ or aMHC-cre;Kcnk $2^{f / f l}$ mice (Figure 6E). These findings suggest that the cardioprotective effects initially observed in global TREK-1-KO mice are mediated by cardiac fibroblasts.

TREK-1 modulates JNK activation. The impaired fibrotic response suggested that TREK-1 might directly influence signaling pathways that dictate cardiac fibrosis development (34). Given the high abundance of fibroblasts in cardiac tissue (34), we investigated changes in MAPKs in whole-heart lysates using hearts from global TREK-1-KO and WT mice after TAC. The MAPK/JNK was differentially phosphorylated in TREK-1-KO mice compared with WT mice after 2 weeks of TAC (Figure 7A). Indeed, hearts from TREK-1-KO mice exhibited attenuation in the phosphorylation of both JNK and the downstream target of JNK, the transcription factor c-Jun (Figure 7A).

Since TREK-1 is known to be stretch sensitive, we tested to determine whether JNK phosphorylation was affected in harvested tissues from TREK-1-KO mice exposed to biomechanical stretch. Using an ex vivo Langendorff preparation, we found that increasing ventricular volumes with balloon inflation in the LV of WT hearts significantly increased whole-heart lysate JNK phosphorylation in comparison with that in perfusion controls, while JNK phosphorylation was attenuated in global TREK-1-KO mice (Figure 7B). We assessed the relative contribution of fibroblasts and myocytes to stretch-induced JNK phosphorylation in response to cardiac ex vivo stretch. Ex vivo cardiac stretch in cre-negative $K c n k 2^{f / f l}$ ventricles induced JNK phosphorylation, which was significantly blunted in $a M H C$-cre;Kcnk $2^{f / f l}$ ventricles (Figure $8 \mathrm{~A}$ ) While ex vivo stretch appeared to have had little effect on wholeheart JNK phosphorylation in $t c f 21-i C r e ; K c n k 2^{f / f l}$ mice (Figure 8A), isolated fibroblasts lacking TREK-1 showed attenuated JNK phosphorylation in response to stimulation by either EGF or TGF- $\beta$ (Figure 8B), suggesting that TREK-1 affects JNK phosphorylation in both cardiomyocytes and fibroblasts. In contrast with the JNK response, activation of ERK1/2 (Supplemental Figure 7A) and p38 $\alpha$ in TREK-1-KO hearts was similar to that in WT in both ex vivo stretch and in vitro ligand stimulation experiments (Supplemental Figure 7B and Supplemental Figure 8).

In addition to these findings, ex vivo stretch in cre-negative $K c n k 2^{f / / f l}$ ventricles was also found to significantly increase the ratio of the proapoptotic protein Bax and antiapoptotic Bcl-2, suggesting enhanced apoptosis (Supplemental Figure 9). This proapoptotic signal was blunted in balloon-stretched $a M H C$-cre;Kcnk $2^{f / / f l}$ ventricles and trended toward reduction in $t c f 21-i \mathrm{Cre} ; \mathrm{Kcnk}^{f / f l f}$, suggesting that the loss of TREK-1 function reduces apoptosis (Supplemental Figure 9). Taken together, these data show that TREK-1 is necessary for the activation of JNK in response to ex vivo biomechanical stretch and in vivo with ventricular loading following TAC. 
A

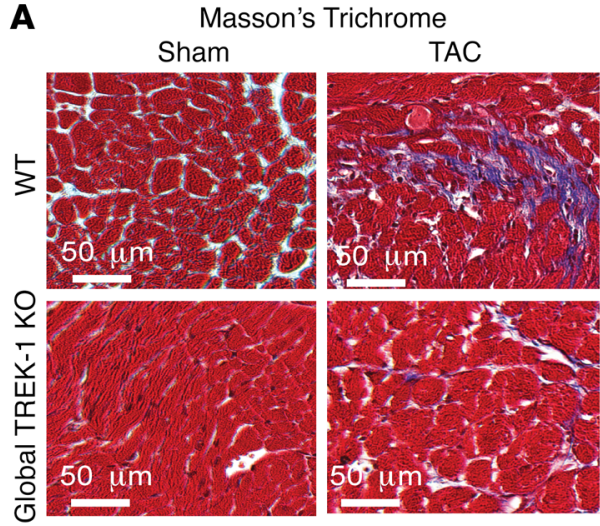

B

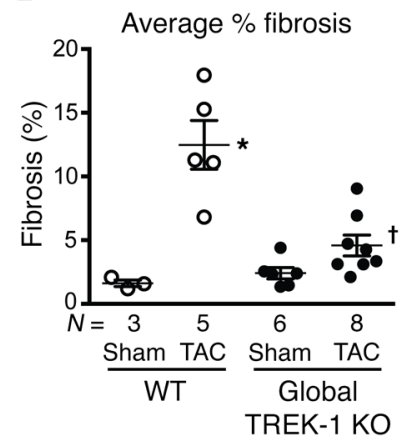

C
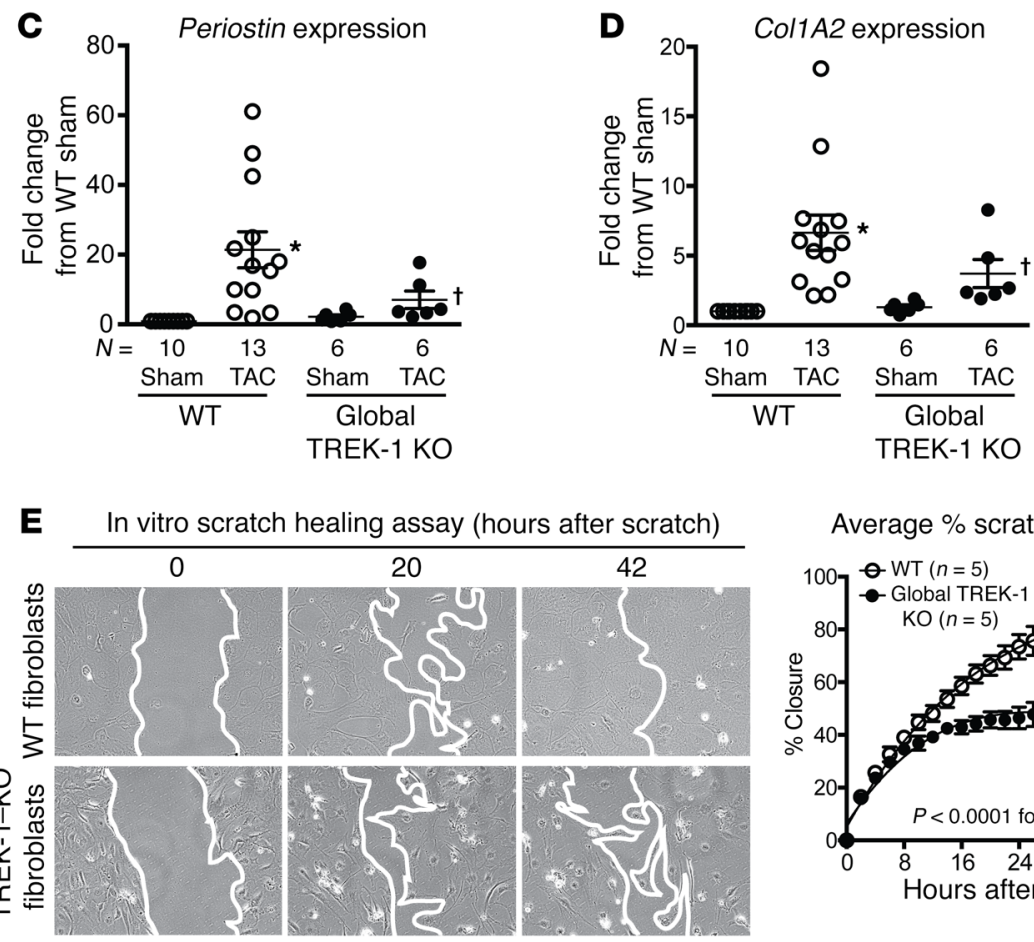

F Cutaneous wound healing (days after surgery)

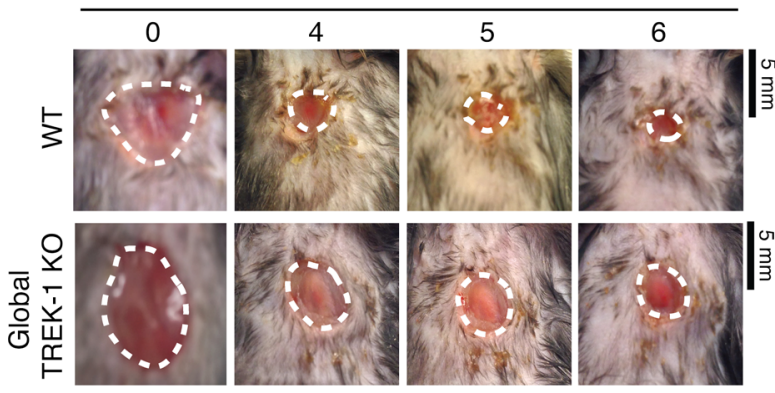

Average $\%$ scratch closure

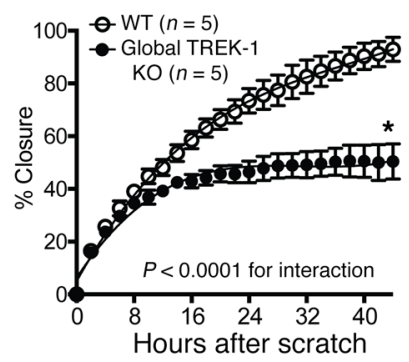

Average $\%$ wound closure

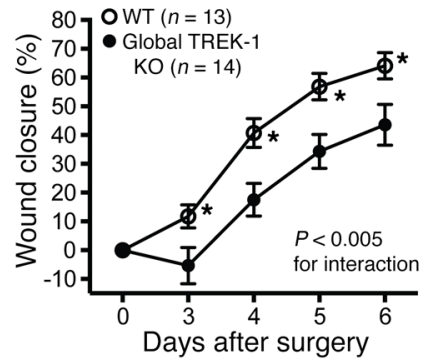

Figure 5. TREK-1 regulates both cardiac and extracardiac fibrosis. (A) Representative Masson's trichrome staining with fibrosis shown in purple. (B) Average percentage fibrosis after 16 weeks of TAC. ${ }^{*} P<0.05$ versus control sham using 1 -sample, 2 -tailed $t$ test. ${ }^{\dagger} P<0.05$ versus WT TAC by Kruskal-Wallis test with Dunn's multiple comparisons test. (C) Average Periostin and (D) collagen type I A2 (Col1A2) gene expression in LVs after 2 weeks of TAC. Statistical comparisons with WT sham, which has a theoretical mean of 1 , were made with a 1-sample, 2-tailed $t$ test. ${ }^{*} P<$ 0.05 versus WT sham. Comparisons among all groups excluding WT sham were made by Kruskal-Wallis test with Dunn's multiple comparisons test. ${ }^{\dagger} P<0.05$ versus WT TAC. (E) Average percentage closure of in vitro scratch closure in WT and TREK-1-KO isolated fibroblasts over 44 hours ( $n=5$ separate experiments). Representative images included below, with leading edge marked in white. Data were compared using 2-way repeated measures ANOVA. ${ }^{*} P<0.05$ in comparison with WT of the same time point using Bonferroni's correction. (F) Average percentage of cutaneous wound closure in control and TREK-1 KO in 6-day wound-healing protocol (WT, $n=13$; TREK-1 KO, $n=14$ ). Representative tracings of wound size were included below. Statistical comparisons between the curves were performed using 2-way repeated measures ANOVA. Scale bars: $5 \mathrm{~mm} .{ }^{*} P<0.05$ in comparison with TREK-1 KO of the same time point using Bonferroni's correction.
Human variation in TREK-1 is associated with abnormal cardiac morphology. To test whether variation in human TREK-1 is associated with variation in cardiac morphology, we investigated the association of SNPs in TREK-1 with cardiovascular phenotypes in the 9,000 patient CATHGEN human cohort. In European Americans, the intronic SNP rs10494995 (minor allele frequency $[\mathrm{MAF}]=0.05)$ and a cluster of linked SNPs were associated with LV septal wall thickness (SWT) (recessive models, all $P<10^{-8}$ ) and
LV hypertrophy (LVH) (recessive models, all $P<0.003)$, with the minor allele associated with more adverse phenotypes (Figure 9). Several intronic SNPs were also significant in African Americans, although the SNPs were different than those in European Americans: $r 4556330$ was associated with left atrial diameter $(\mathrm{MAF}=$ 0.29 , recessive model $P=0.0003)$; rs6540882 was associated with posterior wall thickness $(\mathrm{PWT})(\mathrm{MAF}=0.49$, dominant model $P=$ $0.001)$; rs6665177 was associated with SWT $(\mathrm{MAF}=0.37$, recessive 
A

TREK-1 conditional targeting strategy

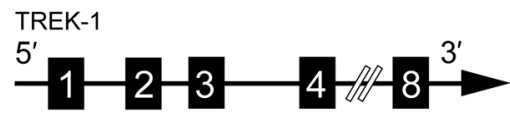

TREK-1 targeting construct $\left(K c n k 2^{f / f f}\right) \quad$ 3'
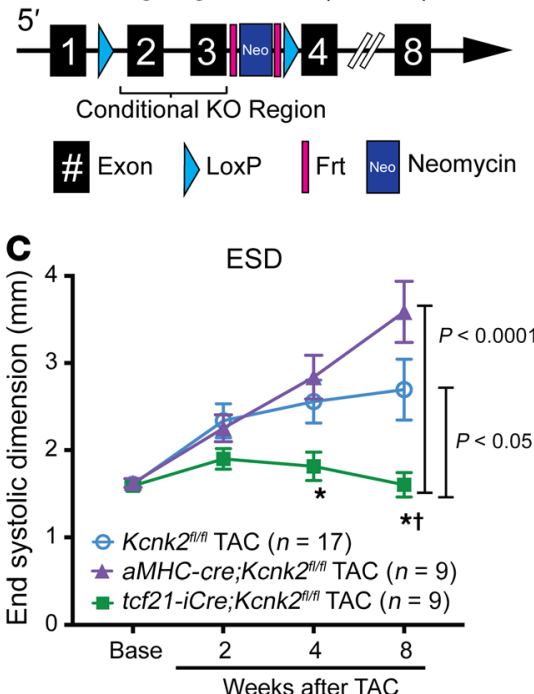

E Fibrosis in conditional myocyte

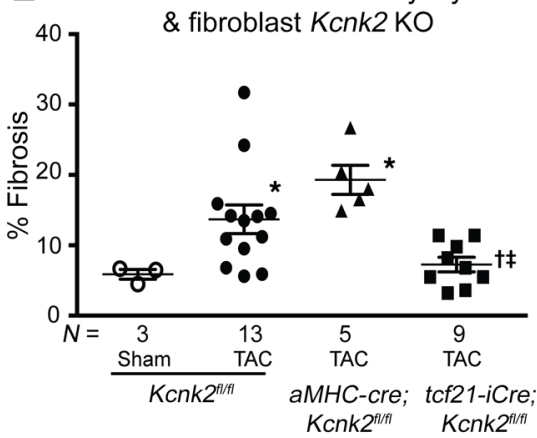

B

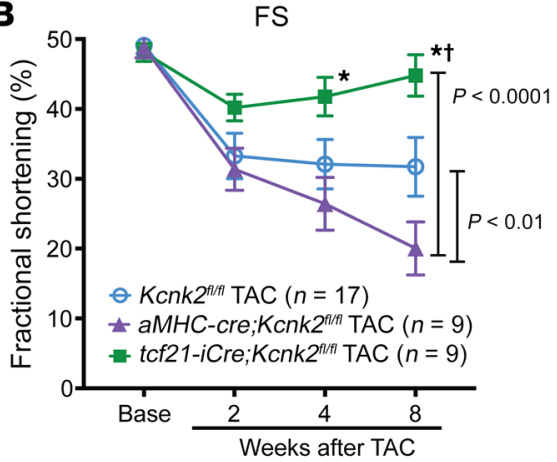

D

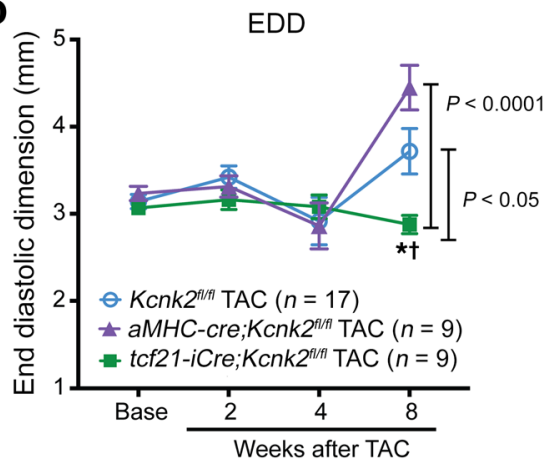

F

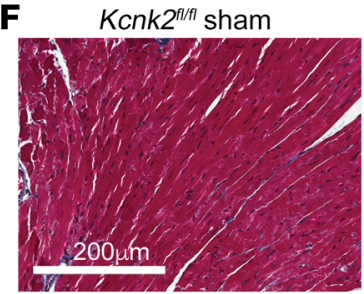

aMHC-cre;Kcnk2 $2^{f / f l}$ TAC

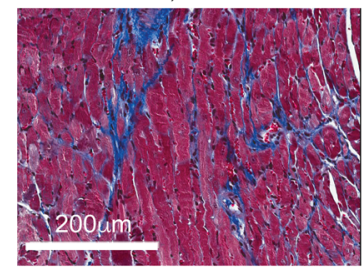

$K c n k 2^{t / f f l} \mathrm{TAC}$

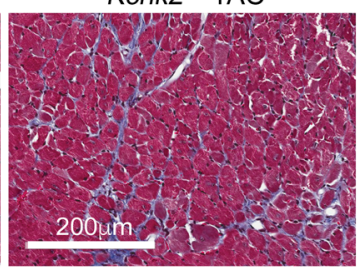

tcf21-iCre;Kcnk2 $2^{\text {fl/f }}$ TAC

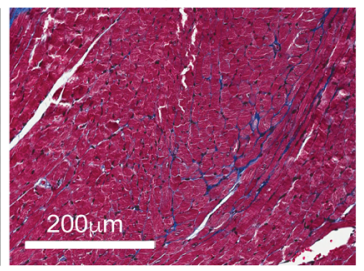

Figure 6. Fibroblast-specific TREK-1 loss of function protects against pressure overload-induced cardiac dysfunction. (A) Conditional TREK-1 targeting strategy schematic: Frt sites flank a neomycin cassette and LoxP sites flank the conditional $\mathrm{KO}$ region. The conditional $\mathrm{KO}$ allele was obtained after Flp-mediated removal of the neomycin selection marker. (B) Serial echocardiographic measurements of average FS, (C) ESD, and (D) EDD in $K c n k 2^{f / f f}$ alone (Cre negative), aMHC-cre;Kcnk $2^{f / / f l}$ (cardiomyocyte specific), and tcf21-iCre; Kcnk $2^{f / f l}$ (fibroblast specific) mice up to 8 weeks after TAC. Error bars reflect SEM. Data were compared using 2-way repeated measures ANOVA. $P$ values for the interaction between genotype and weeks after TAC are shown adjacent to brackets. Comparisons between genotypes at each time point were made using Bonferroni's test for multiple comparisons. ${ }^{\dagger} P$ $<0.05$ versus $K c n k 2^{f l / f l} ;{ }^{*} P<0.05$ versus $a M H C-c r e ; K c n k 2^{f / f f l}$. (E) Tissue fibrosis quantified after 12 weeks of $\mathrm{TAC}$ in $K c n k 2^{f / / f l}$ (sham $=3, \mathrm{TAC}=13$ mice), aMHC-cre-cre; Kcnk $2^{f / f f l}$ (TAC = 5 mice), and tcf21-iCre; Kcnk2f/ffl ( $n=9$ mice) mice using Masson's trichrome stain. (F) Representative histological sections showing fibrosis in purple. Comparisons between genotypes were made by Kruskal-Wallis test with uncorrected Dunn's multiple comparisons test. ${ }^{*} P<0.05$ versus $K c n k 2^{f / f f l}$ sham; ${ }^{+} P<0.05$ versus $K c n k 2^{f l / f f} \mathrm{TAC}$; ${ }^{\ddagger} P<0.05$ versus $a M H C-c r e ; K c n k 2^{f l / f l}$. model $P=0.002$ ); and rs2363561 was associated with LVH (MAF $=0.45$, dominant model, $P=0.0008$ ). While the results for European Americans should be interpreted with caution, given the low allele frequency and small number of influential homozygous individuals, overall these results suggest that genetic loci across human TREK1 are associated with cardiac hypertrophy phenotypes.

\section{Discussion}

To date, the influence of TREK-1 on systolic and diastolic cardiac function or the fibrotic response to augmented hemodynamic load is unknown. Here, we show that TREK-1 and the Drosophila ortholog of TREK-1, sandman, are critical to cardiac function and morphology in both invertebrates and vertebrates. Mice with global deletion of TREK-1 show exaggerated pressure overload-induced cardiac hypertrophy and molecular signatures typical of a pathological cardiac phenotype, yet retain preserved in vivo systolic and diastolic cardiac function. Using both cardiomyocyte-specific and fibroblast-specific TREK-1 loss of function, we show that, in response to in vivo pressure overload, deterioration in cardiac function is only prevented when TREK-1 is deleted specifically in fibroblasts. Finally, we show mechanistically that, under conditions of pressure overload, TREK-1 mediates activation of JNK. We propose a model whereby TREK-1-mediated JNK activation with pressure overload acts to limit excessive cardiomyocyte hypertrophy by dampening hypertrophic signaling pathways, while simultaneously promoting fibroblast migration and proliferation, leading to adverse cardiac remodeling (Figure 10). Our working model is supported by previous work in JNK-KO mice showing enhanced hypertrophy (35), impaired wound healing (36), and impaired fibroblast migration (37). Additionally, deficiency of JNK1 is associated with enhanced deterioration of cardiac function after pressure overload (38), a phenotype similar to what we observed with cardiomyocyte-specific loss of TREK-1 (Figure 6). A critical mechanism responsible for fibrotic responses to injury is myofibroblast 


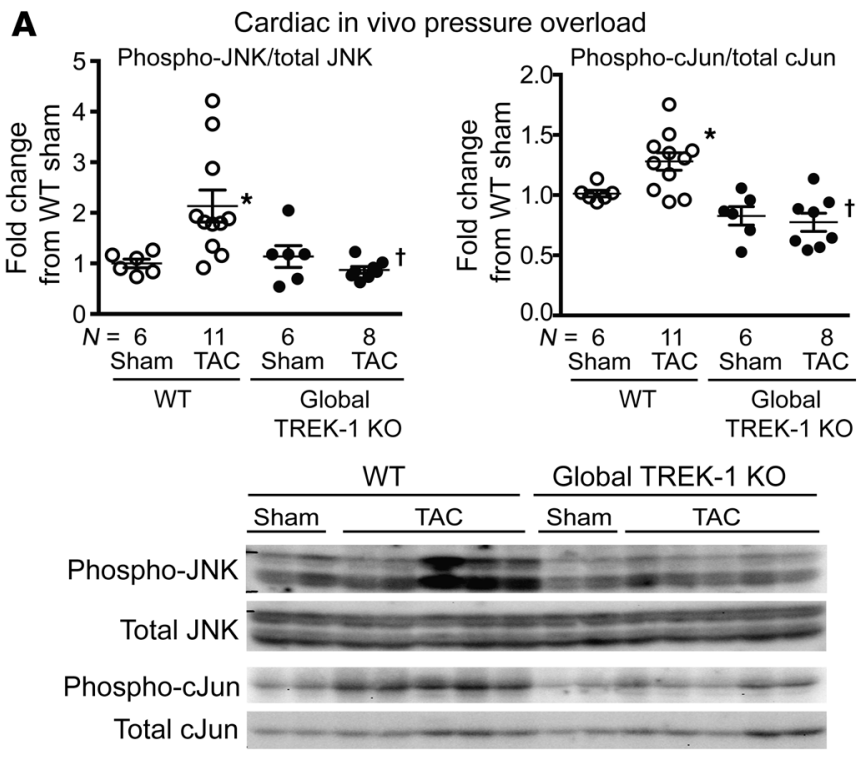

B Cardiac ex vivo stretch: global TREK-1 KO
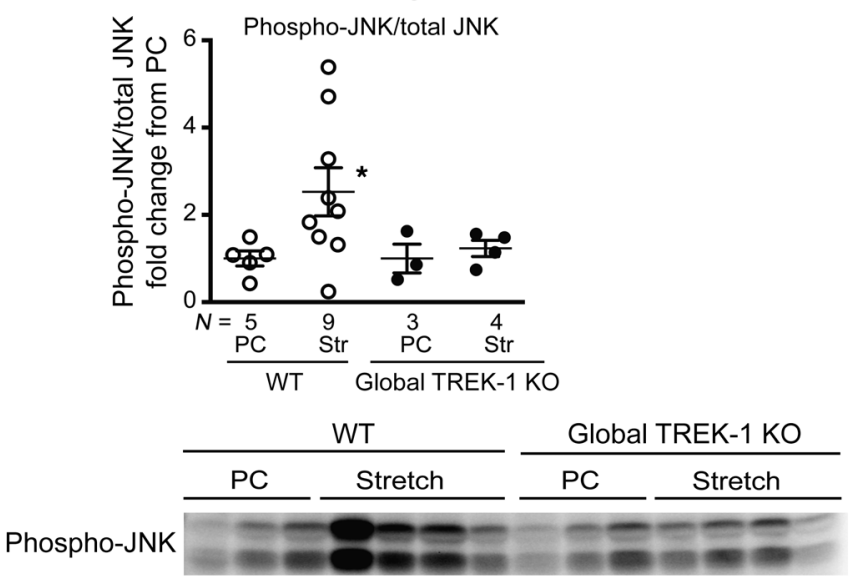

Total JNK

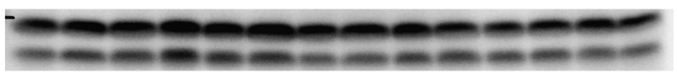

transdifferentiation, which has recently been shown to be affected by p38 $\alpha$ function (39). Interestingly, although TREK-1 affects fibroblast migration and proliferation, the transdifferentiation of fibroblasts to myofibroblasts was unaffected by the loss of TREK-1.

While a great deal is known about the electrophysiologic properties of TREK-1, its role in mammalian cardiac function and response to pressure overload has not previously been described. TREK-1 was first identified almost 2 decades ago as a member of two-pore domain potassium channels, a superfamily of potassium channels that have the topology of 4-transmembrane-spanning domains and two-pore-forming domains in tandem (1). Twopore domain potassium channels, such as TREK-1, are thought to contribute to "background" potassium or "leak" currents and can influence resting membrane potential (2). However, TREK-1 activity is also modulated by mechanical stretch (10), arachidonic acid (40), temperature (41), $\mathrm{pH}$ (42), and G protein-coupled receptor signaling (9). These findings suggest that TREK-1 may have other functions fundamental to cellular homeostasis apart from the maintenance of resting membrane potential. Indeed, TREK-1 has been shown to be involved in endothelial-depen-
Figure 7. Global TREK-1 KO alters JNK phosphorylation. (A) Immunoblotting results for phosphorylated JNK (phospho-JNK) and the downstream JNK target, c-Jun, in WT and TREK-1-KO 3-day and 14-day TAC hearts. Representative array blots shown in the lower panel. (B) Immunoblotting results for phosphorylated JNK in WT and TREK-1-KO hearts harvested after either a perfusion control (PC) or ex vivo stretch (Str). Representative array blots shown in the lower panel. Data for WT sham or perfusion control conditions, which have a theoretical mean of 1 , were compared with Wilcoxon's signed rank test. ${ }^{*} P<0.05$ versus perfusion control condition of the same genotype. Data for all other groups excluding the perfusion control condition were compared by Kruskal-Wallis test with Dunn's multiple comparisons test. ${ }^{\dagger} P<0.05$ versus WT TAC. Full, uncut gels are shown in the supplemental material.

dent vasodilation and nitric oxide production (43). Recent work has shown that TREK-1, via cAMP-dependent interactions with Popeye domain proteins, is important in heart-rate response with exercise (8). These effects were further confirmed by recent work using an independently generated TREK-1 conditional KO, which demonstrated sinoatrial node dysfunction (44). While sinoatrial node function was not assessed in our model, our echocardiography data confirm their findings (44) that $a M H C-c r e ; K c n k 2^{f / f l}$ mice and cre-negative mice have largely similar cardiac function under basal conditions. However, our findings that TREK-1 affects the ventricular diastolic response to injury through its effects on fibrosis raises a new layer of TREK-1-dependent cardiac function modulation that is not dependent on chronotropy.

The hypertrophic phenotype observed in TREK-1-KO mice raises intriguing questions about our current understanding of cardiac hypertrophy and dysfunction. Pressure overload-induced cardiac hypertrophy in TREK-1-KO hearts was associated with reduced $\alpha-\mathrm{MHC}$ and increased $\beta-\mathrm{MHC}$ sarcomeric proteins, a pattern that resembles the fetal gene program. Reexpression of fetal genes is a characteristic finding of pressure overload-induced cardiac hypertrophy and typically precedes the onset of cardiac dysfunction (45). Strikingly, global TREK-1-KO mice exhibit both enhanced concentric hypertrophy and fetal gene activation, yet maintain relatively normal cardiac function. Moreover, increased CAMKII and calcineurin activity, both of which have been associated with deterioration in cardiac function $(46,47)$, were also increased in TREK-1-KO mice with preserved systolic and diastolic function. The preservation of cardiac function in TREK-1-KO mice despite the presence of molecular and genetic signatures commonly associated with cardiac dysfunction suggests (a) that the molecular pathways modulating cardiac function can be separated from pathways that drive pathogenic cardiac remodeling and (b) that fibroblasts are critical participants in response to pressure overload, since specific deletion of TREK-1 from fibroblasts confers cardioprotection.

Our study has particular relevance to diseases such as HFpEF, which is frequently associated with both decreased cardiac compliance and concentric hypertrophy. The development of concentric hypertrophy is often accompanied by extracellular matrix deposition or cardiac fibrosis (48). In experimental models of hypertension, there is an increase in extracellular matrix deposition that occurs early in the development of hypertrophy and is thought to be a beneficial compensation to increased ventricular loading (49). As hypertension persists and concentric hypertrophy devel- 
A

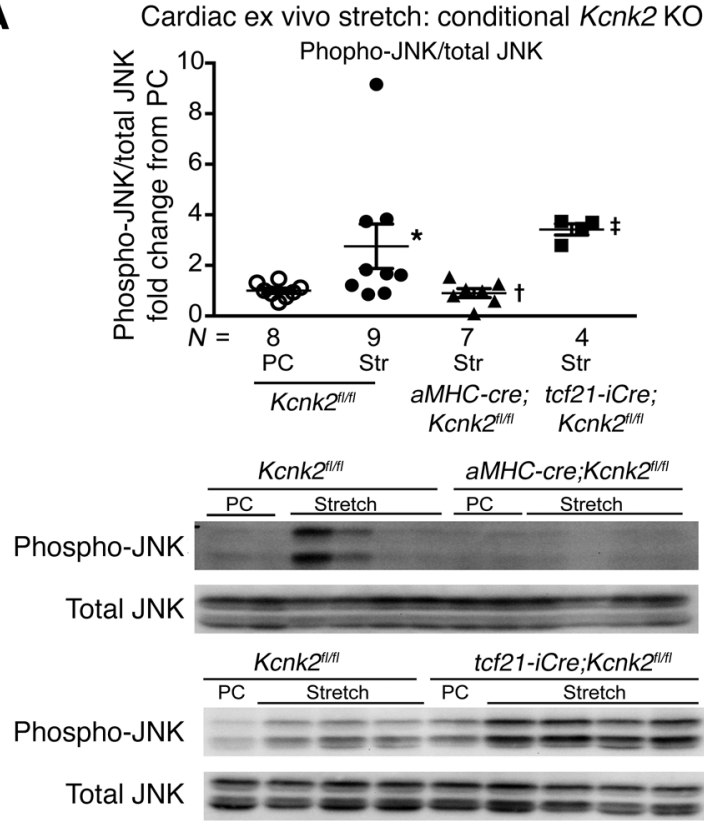

B Isolated fibroblast ligand dose response EGF
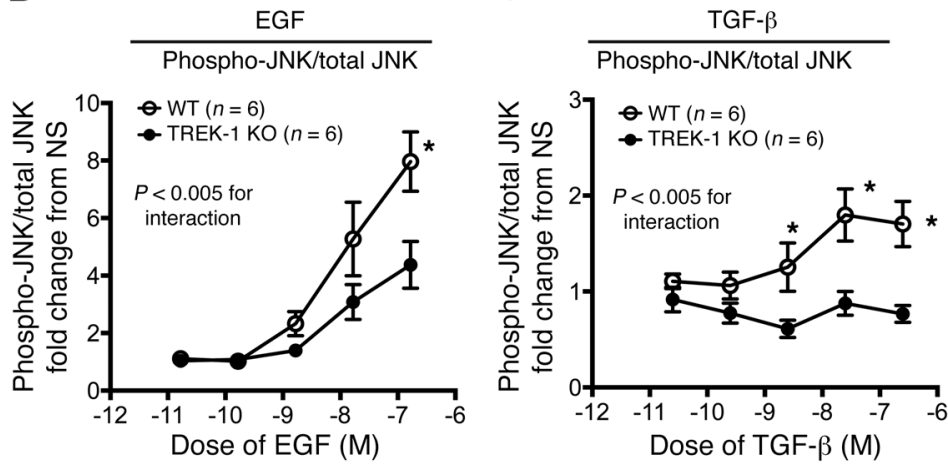

ops, myocyte injury and necrosis are thought to drive additional matrix deposition or "reparative fibrosis" (49). This later increase in cardiac fibrosis is thought to be a major contributor to diastolic dysfunction, although the relative contribution of either cardiac myocytes or cardiac fibrosis remains controversial $(27,50)$. Our work identifying a pathogenic role for fibroblast TREK-1 supports these findings and shows that cardiac fibrosis, not an exaggerated cardiomyocyte hypertrophic response, is the substantial driver of ventricular stiffness. Indeed, these findings may help to explain the relative ineffectiveness of antihypertrophic therapies in conditions frequently associated with concomitant concentric hypertrophy and cardiac fibrosis, such as HFpEF (51). Our findings suggest that therapeutically targeting fibroblast function may be a useful
Figure 8. Cell type-specific TREK-1 KO affects JNK phosphorylation. (A) Immunoblotting results for phosphorylated JNK in Kcnk $2^{f / / f l}$ aMHC-cre;Kcnk $2^{f / f f l}$, and $t c f 21-i C r e ; K c n k 2^{f / f l}$ hearts harvested after either perfusion control or ex vivo stretch. Data are reported as fold change from the perfusion control samples within a gel; all perfusion controls $(n=8)$ for respective genotypes are shown together (Kcnk2 $2^{f / f l}, n=4$; aMHC-cre; Kcnk $2^{f / / f l}, n=3$; and tcf21-iCre; $\left.K c n k 2^{f / f l}, n=1\right)$. Data for perfusion control conditions, which have a theoretical mean of 1 , were compared with Wilcoxon's signed rank test. ${ }^{*} P<0.05$ versus perfusion control conditions of the same genotype. Data for all other groups excluding perfusion control conditions were compared by Kruskal-Wallis test with Dunn's multiple comparisons test. ${ }^{\dagger} P<0.05$ versus Kcnk $2^{f l / f l}$ TAC; ${ }^{\ddagger} P<0.05$ versus aMHC-cre;Kcnk2 $2^{f / f l}$. (B) Average JNK phosphorylation/total JNK in response to 20 minutes of treatment with EGF and 40 minutes of treatment with TGF- $\beta$ in isolated WT and global TREK-1-KO lung fibroblasts ( $n=6$ separate experiments). Doses of either EGF or TCF- $\beta$ were tested with a range of concentrations from $10^{-11} \mathrm{M}$ to $10^{-7} \mathrm{M}$. Data are expressed as fold change from a nonstimulated (NS) condition. Data compared by 2-way ANOVA with Bonferroni's multiple comparisons test. ${ }^{*} P<0.05$ versus TREK-1-KO fibroblasts. Full, uncut gels are shown in the supplemental material.

approach to improving cardiac mechanics under disease conditions that promote diastolic dysfunction.

In summary, we show that the loss of function of the Drosophila potassium channel sandman results in impaired cardiac function in the fly. In mammals, global deletion of the stretch-dependent mammalian ortholog of sandman, TREK-1, prevents the development of systolic and diastolic dysfunction in response to pressure overload despite a phenotype of exaggerated cardiomyocyte hypertrophy and activation of genetic and molecular signatures typically associated with a pathological cardiac state. The influence of TREK-1 on cardiac function is largely driven by its presence in cardiac fibroblasts and its effect on fibroblast function, since conditional deletion of TREK- 1 in fibroblasts prevents the functional decline associated with pressure overload injury. This uncoupling of cardiac remodeling and dysfunction is associated with blunted activation of JNK/MAPK in the heart, though additional studies will be required to determine its precise role in promoting cardioprotection in the context of TREK-1 deletion. The relevance of this work to human populations is supported by findings of gene polymorphisms in humans with cardiac hypertrophy, although the effect of these polymorphisms on TREK- 1 function will need to be addressed in future studies. Taking these data together, our study identifies a role of TREK-1 on cardiac function and provides insights into how cardiac fibroblasts can influence overall ventricular function under pathogenic conditions.

\section{Methods}

Drosophila stocks. Genomic deletion stocks (Df[2R]Exel7094, Df[2R] Exel6055, Df[2R]Exel7095), piggyBac insertion stocks (PBac[RB]sandman e00867), and Actin5C-Gal4 stocks were obtained from the Bloomington Drosophila Stock Center. All stocks were maintained on standard yeast protein media at room temperature. The tinC-Gal4 stock was provided by Manfred Frasch (Mount Sinai Medical Center, New York, New York, USA) (52). 
A

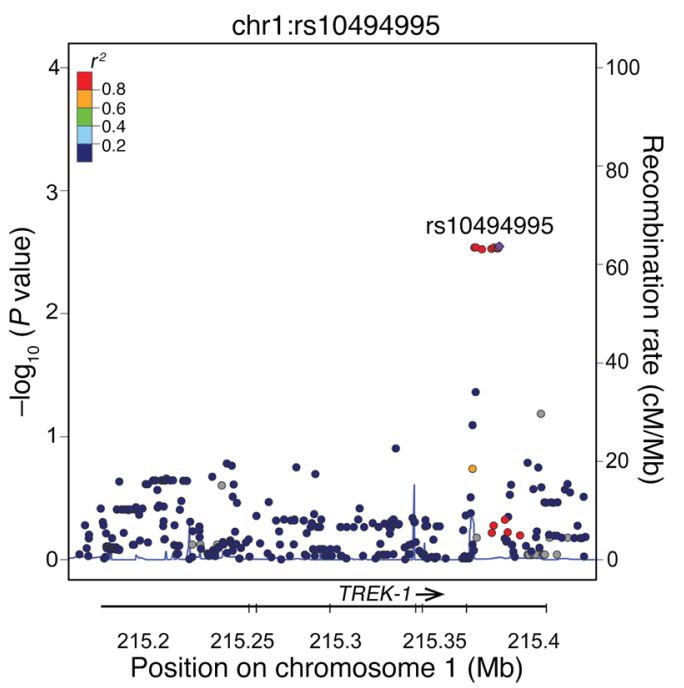

B

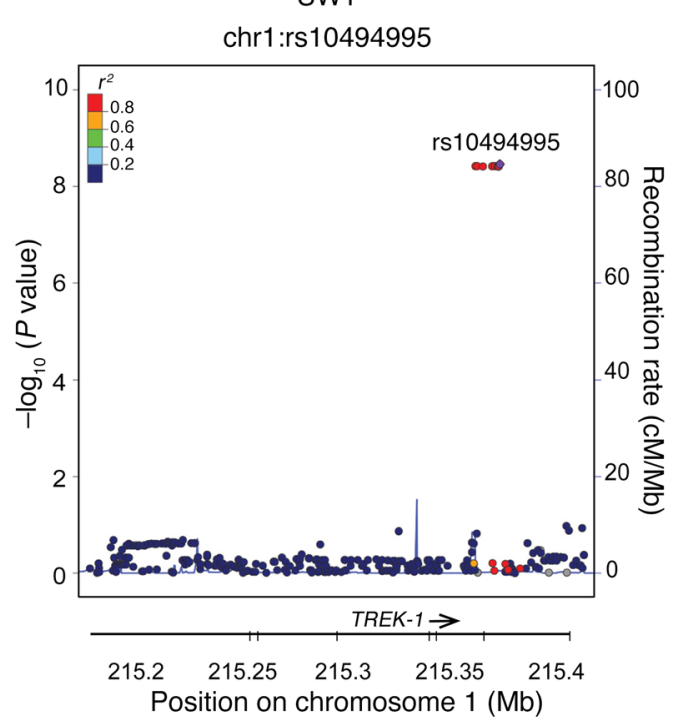

Figure 9. TREK-1 SNP

associated with human cardiac hypertrophy. Locus zoom plots SNP rs10494995 in TREK-1 is associated with both (A) LVH and (B) SWT assessed by echocardiography.
Generation of transgenic Drosophila. The cDNA encoding sandman were isolated by reverse transcription PCR (RT-PCR) from w1118 flies, subcloned into p-UAST, and verified by sequencing. Transgenic Drosophila harboring sandman under the control of upstream activating sequence (UAS) were generated by established methods (53). Organ-specific expression of sandman was accomplished by genetically crossing UAS-sandman flies with those expressing tinC-gal4 (tinC $>$ sandman, cardiac specific expression) or Actin5C-Gal4 (actin $>$ sandman, ubiquitous expression).

Drosophila cardiac measurements using OCT. Cardiac function in adult Drosophila was measured using a custom-built optical coherence tomography (OCT) microscopy system (Bioptigen Inc.), as previously described (54). Briefly, adult female Drosophila between 7 and 10 days after eclosion were briefly subjected to $\mathrm{CO}_{2}$, placed on a soft gel support, and allowed to fully awaken based on body movement. OCT M-modes were recorded, and images were processed using ImageJ software (NIH) using a $125 \mu \mathrm{m}$ standard. End-diastolic dimension (EDD) and end-systolic dimension (ESD) were determined from 3 consecutive heartbeats. Heart rate was determined by counting the total number of beats that occurred during a 2.6-second or 5.2-second recording and calculating bpm. FS was calculated as follows: (EDD ESD) $/$ EDD $\times 100$. FS was a calculation of the percentage change in cardiac chamber dimensions during contraction, and a decrease in FS was interpreted as a reduction in systolic function.

Experimental animals. Eight- to twelve-week-old control C57BL/6 WT mice and global TREK-1-KO mice were used for this study. TREK-1KO mice were provided by Robert Bryan (Baylor College of Medicine, Houston, Texas, USA) (55). For in vivo experiments, at least 4 animals were used to assess either a noninvasive or invasive parameter.

Generation of pressure overload and serial echocardiography. Pressure overload in mice was induced using methods previously described (32). Serial echocardiography was performed on conscious mice from all groups with a Vevo 2100 high-resolution imaging system (VisualSonics), as previously described. Mice underwent either a sham or pressure overload procedure in a nonrandomized fashion. Two separate readers read all echocardiograms in an unblinded manner, and both read- ings were averaged. Mice with an ineffective TAC, defined as either a hemodynamically measured TAC gradient of less than $15 \mathrm{mmHg}$ or an increase in calculated LV mass by echocardiography of less than $10 \%$ from baseline, were excluded. Mice were excluded in the following instances: 16 weeks of TAC experiment shown in Figure 2, 1 WT mouse excluded; 8 weeks of conditional TAC shown in Figure 5 and Supplemental Table 4, 2 Kcnk $2^{f / f l}, 1$ aMHC-cre;Kcnk $2^{f / f l}$ mice excluded.

Pressure-volume loop analysis. In vivo pressure-volume analysis was performed as previously described (56). Of note, in animals that had undergone TAC, the ligature in the transverse aorta was carefully cut prior to pressure-volume loop generation. Subsequently, parallel conductance $(\mathrm{Vp})$ was determined by $10 \mu$ injection of $15 \%$ saline into the right jugular vein to establish the Vp of the blood pool. The derived Vp was used to correct the pressure-volume loop data. Data were recorded digitally at $1,000 \mathrm{~Hz}$ and analyzed with pressure-volume analysis software (PVAN data analysis software version 3.3; Millar Instruments), as previously described (56). Mice that died after receiving anesthesia or became hypotensive during the course of the protocol, suggesting a surgical complication, were excluded.

Histologic analyses. Freshly harvested cardiac samples were placed in sucrose/PBS solution at $4^{\circ} \mathrm{C}$ for 2 to 4 hours, placed in cross-section in OCT (Miles Pharmaceuticals), and snap-frozen in liquid nitrogen. Slides from frozen sections were subsequently fixed in $4 \%$ paraformaldehyde and stained with H\&E. Myocyte cross-sectional area was measured by wheat germ agglutinin staining on frozen sections. Fibrosis was measured using Masson's trichrome stain. Myocyte crosssectional area was obtained using ImageJ software. Fibrosis was quantified using Aperio ImageScope software.

Wound-healing assay. The dermal wound-healing assay was performed as previously described (57). In brief, $6 \mathrm{~mm}$ wounds were created with a disposable biopsy punch (Integra Miltex) on the dorsal midline of anesthetized mice. Wounds were traced on transparent plastic sheets and measured to assess wound retraction and health. Wound tracings were analyzed by Image J software to measure surface areas.

Scratch assay. The fibroblast wound-healing assay was performed in isolated lung fibroblasts obtained from TREK-1-KO and WT mice. Fibro- 


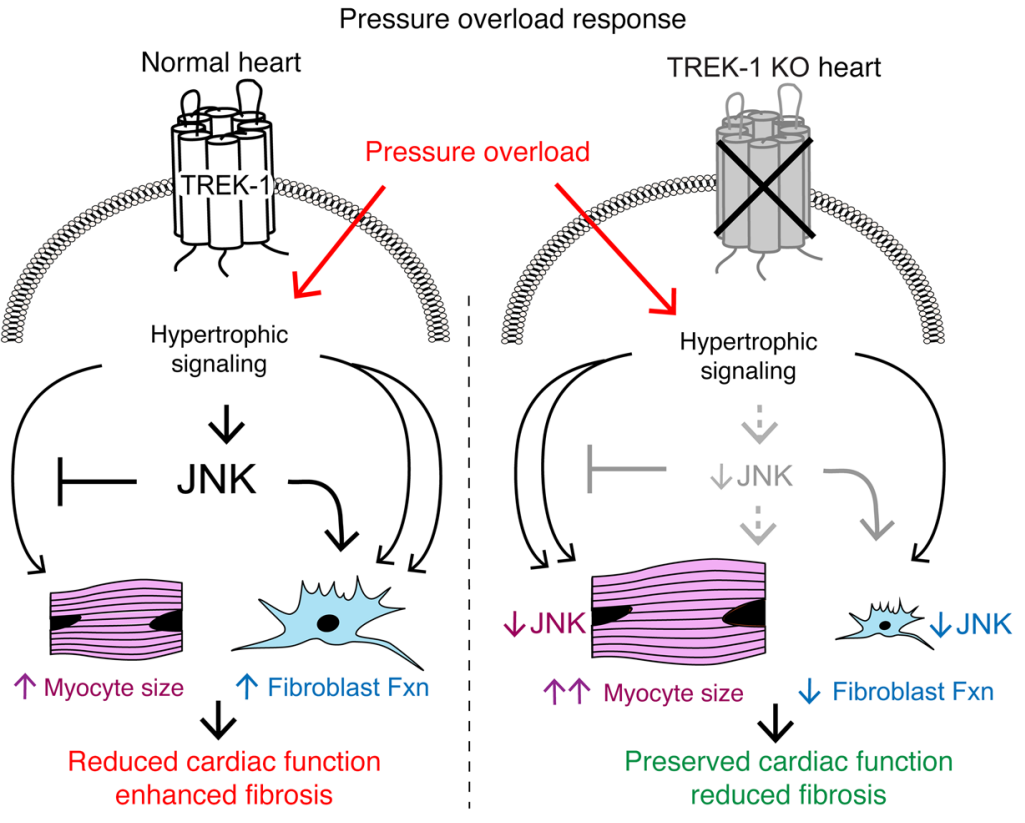

Figure 10. Effect of TREK-1 on cardiac remodeling and function. Working model of TREK-1's influence on cardiac morphology and function. Under normal conditions (left side), pressure overload stimulates TREK-1-mediated JNK phosphorylation, which both inhibits concentric hypertrophy and activates fibroblast function, resulting in cardiac dysfunction. In TREK-1 KO (right panel), pressure overloadmediated JNK phosphorylation is inhibited, resulting in enhanced concentric hypertrophy and reduced fibroblast function that is overall cardioprotective. blasts of each genotype were plated on a 6-well dish that was coated with poly-D-lysine (Millipore). Cells were starved in serum-free medium for 48 hours prior to scratch. A scratch was applied in each well, and serial images were obtained at $37^{\circ} \mathrm{C}$ on a Zeiss Axio Observer inverted microscope with stage incubator, $\mathrm{CO}_{2}$ buffering, and outer environmental chamber. A CoolSNAP ES2 camera was used for acquisition, and the system was controlled by MetaMorph, which captured pictures at a $\times 10$ objective every 2 hours. Percentage of closure was determined by (a) inscribing a region of interest over the initial scratch area and (b) measuring the area from the leading edge within the region of interest. Two separate readers analyzed serial images using ImageJ software, and the data were averaged.

Myocyte-fibroblast fractionation. LVs were harvested from mice and digested and resuspended in $2 \mathrm{ml}$ dispersion media. The cell suspension was transferred to a $15 \mathrm{ml}$ centrifugation tube on top of discontinuous Percoll gradients. After 45 minutes at $1800 \mathrm{~g}$ centrifugation, the myocytes and fibroblasts were separated as previously described (58).

Myofibroblast transdifferentiation and fibroblast dose response. Primary fibroblast cultures were obtained from lung tissue from either adult WT or TREK-1-KO mice that were 8 to 10 weeks of age. Lung tissue was minced into $1 \mathrm{~mm}$ pieces in $0.5 \mathrm{ml}$ PBS. Minced tissue was then digested in an enzyme buffer containing collagenase (Roche) and DNAase I (Roche) and incubated at $37^{\circ} \mathrm{C}$ for 15 to 20 minutes. Cells were then transferred to a Falcon tube containing $5 \mathrm{ml}$ of ice-cold FBS. The remaining cell suspensions were passed through a $70 \mu \mathrm{m}$ mesh nylon and centrifuged at $478 g$ for 5 minutes; the supernatant was then aspirated. Fibroblast suspensions were then resuspended in $2 \mathrm{ml} \mathrm{DMEM}$ with 15\% FBS and penicillin/streptomycin and plated. Both myofibroblast transdifferentiation and ligand dose-response studies were performed on lung fibroblasts after 3 to 4 passages. Myofibroblast transdifferentiation was assessed using previously published methods (57). In brief, cultured lung fibroblasts were treated with either vehicle or 10 $\mathrm{ng} / \mathrm{ml} \mathrm{TGF-} \beta$. After 48 hours, cells were fixed in $4 \%$ paraformaldehyde. Fibroblasts were incubated for 1.5 hours at room temperature with SMA antibody (1:1000, mouse monoclonal, MilliporeSigma), as previously described (57). Ligand dose-response experiments were conducted on lung fibroblasts plated on a 6-well cell-culture dish. Fibroblasts were serum starved overnight and then exposed to either epidermal growth factor (EGF) for 20 minutes or TGF- $\beta$ for 40 minutes.

Ex vivo cardiac stretch. Ex vivo cardiac stretch was performed using previously published methods (59). An adequate cardiac stretch was defined as achieving an EDD pressure greater than $20 \mathrm{mmHg}$ that was sustained for 30 minutes. One $K c n k 2^{f / f l}$ (cre negative) mouse was excluded due to inadequate cardiac stretch.

Gene expression analysis. At study termination, hearts were snap-frozen in liquid nitrogen and stored at $-80^{\circ} \mathrm{C}$. RNA samples were prepared from LV tissue and were extracted using the RNeasy Microarray Tissue Mini Kit (QIAGEN) according to the manufacturer's instructions. mRNA expression of selected genes was carried out by real-time quantitative RT-PCR with a Bio-Rad CFX96 machine. All reactions included a GAPDH internal standard.

Immunoblotting. Tissue samples were homogenized in Nonidet P-40 (NP-40) lysis buffer containing $20 \mathrm{mM}$ Tris (pH 7.4), $137 \mathrm{mM}$ $\mathrm{NaCl}, 1 \% \mathrm{NP}-40,20 \%$ glycerol, $10 \mathrm{mM}$ PMSF, $1 \mathrm{mM} \mathrm{Na}_{3} \mathrm{VO}_{4}, 10 \mathrm{mM}$ $\mathrm{NaF}$, aprotinin $(2.5 \mathrm{mg} / \mathrm{ml})$, and leupeptin $(2.5 \mathrm{mg} / \mathrm{ml})$. Protein concentrations were assayed with Bio-Rad protein assay reagent, and 80-100 $\mu \mathrm{g}$ protein was denatured by heating at $95^{\circ} \mathrm{C}$ for 5 minutes before resolving by SDS-PAGE. The following dilutions of primary antibody were used: phospho-JNK (1:800; catalog V7931, Promega); total JNK (1:1,000; catalog sc-571, Santa Cruz Biotechnology Inc.); Bax (1:1,000; catalog 2772, Cell Signaling Technology); Bcl-2 (1:1,000; catalog 2872, Cell Signaling Technology); phospho-p38 $\alpha$ (1:1,000; catalog 9211, Cell Signaling Technology); total phospho-p38 $\alpha$ (1:1,000; catalog 9212, Cell Signaling Technology); phospho-SMAD3 (1:800; catalog ab52903, Abcam); and total SMAD2/3 (1:1,000; catalog 3102, Cell Signaling Technology). Detection was carried out by an enhanced chemiluminescence detection system (Amersham Biosciences). Densitometric analysis was performed with Bio-Rad Fluor-S MultiImager software.

Conditional TREK-1 mouse creation. The conditional TREK-1-KO (Kcnk2) mouse was generated by Cyagen Biosciences. TREK-1 was targeted for homologous recombination in C57BL/6 embryonic stem 
cells, with loxP sites introduced before exon 2 and after exon 3 (Figure $5 \mathrm{~A}$ ), and a loss-of-function $m K c n k 2$ gene was generated by introducing a frameshift mutation. To engineer the targeting vector, the $5^{\prime}$ homology arm, 3' homology arm, and conditional Kcnk2-KO region were amplified from BAC DNA and confirmed by end sequencing. In the targeting vector, Frt sites flanked the Neo cassette and LoxP sites flanked the conditional KO region. The conditional KO allele was obtained after Flp-mediated removal of the Neo selection marker. Cardiac-specific Kcnk2-KO mice were generated by crossing the Kcnk2 $2^{f / f l}$ mice with $a M H C$-cre mice. Cardiomyocyte Kcnk2 KO was confirmed by real-time PCR to assess Kcnk2 in cardiomyocyte preparations (Supplemental Figure 10). Fibroblast-specific Kcnk2 KO was achieved by crossing the conditional Kcnk2 KO with tcf21-inducible cre recombinase-expressing mice ( $t c f 21-i \mathrm{Cre}$ ), provided by Michelle Tallquist (University of Hawaii, Honolulu, Hawaii, USA) (60). Tcf21-iCre expression was induced by injecting tamoxifen i.p. into $t c f 21-i \mathrm{Cre} ; \mathrm{Kcnk} 2^{f l / f l}$ mice for 10 days. All mouse strains were on the C57BL/6 background.

Variation in human TREK1. The study population for the human genetic association study was from the CATHGEN study, which consists of individuals recruited sequentially through the cardiac catheterization laboratories at Duke University Medical Center (61). SNP data were obtained from GWAS (Illumina Omni1-Quad Infinium Bead Chip) (62). SNPs within and $5 \mathrm{~kb}$ upstream and downstream from human TREK1 were analyzed (74 directly genotyped and 304 imputed SNPs in the European Americans and 82 directly genotyped and 310 imputed SNPs in African Americans). Cardiovascular phenotypes analyzed included the following: systolic blood pressure $(n=2313), \mathrm{LV}$ ejection fraction $(n=$ 2862), and echocardiographic variables $(n=993$, left atrial diameter, LV PWT, ventricular SWT, and LVH [defined as an ordinal trait with 4 categories from none to severe, $n=1143]$ ). Multivariable linear regression with additive, dominant, and recessive models of risk, adjusted for age, sex, hypertension, diabetes status, smoking status, number of diseased vessels, and body mass index were used for analysis (systolic blood pressure was not adjusted for hypertension). Significance was defined as $\alpha=0.05$ unadjusted for multiple comparisons. Analyses were performed using SAS V9.4. All SNPs were in Hardy-Weinberg equilibrium.

Statistics. Data are summarized as mean \pm SEM. Statistical analysis was performed with the GraphPad Prism Software Package, version 6.0 (GraphPad Inc). In comparisons performed against a sham condition (with a hypothetical value of 1), a 1-sample, 2 tailed $t$ test or Wilcoxon's signed rank test was used. Comparisons between 2 groups that were not normally distributed were made using Mann-Whitney $U$ test with 2-tailed $P$ value. When more than 2 groups were compared, a ShapiroWilk normality test was performed to assess for normal distribution. If parameters passed the normality test, 1-way ANOVA was used; if parameters did not pass the test, Kruskal-Wallis test with Dunn's multiple comparisons test was used. When serial measurements between 2 groups were compared, 2-way repeated measures ANOVA with Bonferroni's multiple comparisons test was used, unless otherwise specified. $P<0.05$ was considered statistically significant. Error bars reflect SEM.

Study approval. Research with animals carried out for this study was handled according to approved protocols and animal welfare regulations of the Duke University Medical Center's institutional review boards.

\section{Author contributions}

DMA, TEL, LJW, SF, GSP, SHS, MJW, and HAR participated in experimental design and interpretation of the data. DMA, TEL, LJW, SF, LM, GC, and HGW performed experiments. DMA, TEL, LM, LJW, SHS, HGW, and SF analyzed experiments. DMA, TEL, LJW, LM, GC, HGW, SF, GSP, SHS, MJW, and HAR wrote or edited the manuscript.

\section{Acknowledgments}

We sincerely thank Robert Bryan (Baylor College of Medicine) for providing the global TREK-1-KO mice. Requests for these mice should be addressed to him. We would also like to thank Wei Duan, Jonne Woodard, and Zhiqiang Chen for technical assistance in the completion of experiments. This work was supported by NIH grant 1K08HL125905-01 (to DMA) and NIH grants HL56687 and HL075443 (to HAR).

Address correspondence to: Howard A. Rockman, Duke University Medical Center, DUMC Box 3104, Room 226, CARL Building, Durham, North Carolina 27710, USA. Phone: 919.668.2521; Email:h.rockman@duke.edu.

LJW's present address is: Kentucky College of Osteopathic Medicine, University of Pikeville, Pikeville Kentucky, USA.

MJW's present address is: Department of Medicine, University of Virginia, Charlottesville, Virginia, USA.

HGW and GSP's present address is: Department of Medicine, Weill Cornell Medical Center New York, New York, USA.
1. Fink $\mathrm{M}$, et al. Cloning, functional expression and brain localization of a novel unconventional outward rectifier $\mathrm{K}+$ channel. EMBO J. 1996;15(24):6854-6862.

2. Goonetilleke L, Quayle J. TREK-1 K(+) channels in the cardiovascular system: their significance and potential as a therapeutic target. Cardiovasc Ther. 2012;30(1):e23-e29.

3. Schmidt C, et al. Stretch-activated two-poredomain (K2P) potassium channels in the heart: Focus on atrial fibrillation and heart failure. Prog Biophys Mol Biol. 2017;130(Pt B):233-243.

4. Gurney A, Manoury B. Two-pore potassium channels in the cardiovascular system. Eur Biophys J. 2009;38(3):305-318.

5. Kim D, Clapham DE. Potassium channels in car- diac cells activated by arachidonic acid and phospholipids. Science. 1989;244(4909):1174-1176.

6. Xian Tao L, et al. The stretch-activated potassium channel TREK-1 in rat cardiac ventricular muscle. Cardiovasc Res. 2006;69(1):86-97.

7. Hund TJ, et al. $\beta(\mathrm{IV})$-Spectrin regulates TREK-1 membrane targeting in the heart. Cardiovasc Res. 2014;102(1):166-175.

8. Froese A, et al. Popeye domain containing proteins are essential for stress-mediated modulation of cardiac pacemaking in mice. JClin Invest. 2012;122(3):1119-1130.

9. Murbartián J, Lei Q, Sando JJ, Bayliss DA. Sequential phosphorylation mediates receptor- and kinase-induced inhibition of TREK-1 background potassium channels. J Biol Chem.
2005;280(34):30175-30184.

10. Patel AJ, et al. A mammalian two pore domain mechano-gated S-like $\mathrm{K}+$ channel. EMBO J. 1998;17(15):4283-4290.

11. Klassen MP, Peters CJ, Zhou S, Williams HH, Jan LY, Jan YN. Age-dependent diastolic heart failure in an in vivo Drosophila model. Elife. 2017;6:e20851.

12. Owan TE, Hodge DO, Herges RM, Jacobsen SJ, Roger VL, Redfield MM. Trends in prevalence and outcome of heart failure with preserved ejection fraction. N Engl J Med. 2006;355(3):251-259.

13. Katz DH, Beussink L, Sauer AJ, Freed BH, Burke MA, Shah SJ. Prevalence, clinical characteristics, and outcomes associated with eccentric versus concentric left ventricular hypertrophy in heart failure with preserved ejection fraction. Am J 
Cardiol. 2013;112(8):1158-1164.

14. Zile MR, Lewinter MM. Left ventricular enddiastolic volume is normal in patients with heart failure and a normal ejection fraction: a renewed consensus in diastolic heart failure. J Am Coll Cardiol. 2007;49(9):982-985.

15. Maurer MS, Burkhoff D, Fried LP, Gottdiener J, King DL, Kitzman DW. Ventricular structure and function in hypertensive participants with heart failure and a normal ejection fraction: the Cardiovascular Health Study. J Am Coll Cardiol. 2007;49(9):972-981.

16. Edvardsen T, et al. Regional diastolic dysfunction in individuals with left ventricular hypertrophy measured by tagged magnetic resonance imaging--the Multi-Ethnic Study of Atherosclerosis (MESA). Am Heart J. 2006;151(1):109-114.

17. Fox ER, et al. Left ventricular geometric patterns in the Jackson cohort of the Atherosclerotic Risk in Communities (ARIC) Study: clinical correlates and influences on systolic and diastolic dysfunction. Am Heart J. 2007;153(2):238-244.

18. Marks AR. Calcium cycling proteins and heart failure: mechanisms and therapeutics. JClin Invest. 2013;123(1):46-52.

19. Kawaguchi M, Hay I, Fetics B, Kass DA. Combined ventricular systolic and arterial stiffening in patients with heart failure and preserved ejection fraction: implications for systolic and diastolic reserve limitations. Circulation. 2003;107(5):714-720.

20. Lam CS, et al. Cardiac structure and ventricular-vascular function in persons with heart failure and preserved ejection fraction from Olmsted County, Minnesota. Circulation. 2007;115(15):1982-1990.

21. Zile MR, Baicu CF, Gaasch WH. Diastolic heart failure--abnormalities in active relaxation and passive stiffness of the left ventricle. $N$ EnglJ Med. 2004;350(19):1953-1959.

22. Bronzwaer JG, Paulus WJ. Matrix, cytoskeleton, or myofilaments: which one to blame for diastolic left ventricular dysfunction? Prog Cardiovasc Dis. 2005;47(4):276-284.

23. Weber KT, Brilla CG. Pathological hypertrophy and cardiac interstitium. Fibrosis and reninangiotensin-aldosterone system. Circulation. 1991;83(6):1849-1865.

24. Thannickal VJ, Zhou Y, Gaggar A, Duncan SR. Fibrosis: ultimate and proximate causes. JClin Invest. 2014;124(11):4673-4677.

25. Anderson KP, Walker R, Urie P, Ershler PR, Lux RL, Karwandee SV. Myocardial electrical propagation in patients with idiopathic dilated cardiomyopathy. JClin Invest. 1993;92(1):122-140.

26. Schwartzkopff B, Brehm M, Mundhenke M, Strauer BE. Repair of coronary arterioles after treatment with perindopril in hypertensive heart disease. Hypertension. 2000;36(2):220-225.

27. Yamamoto K, et al. Myocardial stiffness is determined by ventricular fibrosis, but not by compensatory or excessive hypertrophy in hypertensive heart. Cardiovasc Res. 2002;55(1):76-82.

28. Gulati A, et al. Association of fibrosis with mortality and sudden cardiac death in patients with nonischemic dilated cardiomyopathy. JAMA. 2013;309(9):896-908.

29. Wong TC, et al. Myocardial extracellular volume fraction quantified by cardiovascular magnetic resonance is increased in diabetes and associated with mortality and incident heart failure admission. Eur Heart J. 2014;35(10):657-664.

30. Pellman J, Zhang J, Sheikh F. Myocyte-fibroblast communication in cardiac fibrosis and arrhythmias: Mechanisms and model systems. J Mol Cell Cardiol. 2016;94:22-31.

31. Pimentel D, Donlea JM, Talbot CB, Song SM, Thurston AJF, Miesenböck G. Operation of a homeostatic sleep switch. Nature. 2016;536(7616):333-337.

32. Rockman HA, et al. Segregation of atrial-specific and inducible expression of an atrial natriuretic factor transgene in an in vivo murine model of cardiac hypertrophy. Proc Natl Acad Sci US A. 1991;88(18):8277-8281.

33. Kakkar R, Lee RT. Intramyocardial fibroblast myocyte communication. Circ Res. 2010;106(1):47-57.

34. Deb A, Ubil E. Cardiac fibroblast in development and wound healing. JMol Cell Cardiol. 2014;70:47-55.

35. Liang Q, Bueno OF, Wilkins BJ, Kuan CY, Xia Y, Molkentin JD. c-Jun N-terminal kinases (JNK) antagonize cardiac growth through cross-talk with calcineurin-NFAT signaling. EMBO J. 2003;22(19):5079-5089.

36. Schumacher M, et al. Efficient keratinocyte differentiation strictly depends on JNK-induced soluble factors in fibroblasts. J Invest Dermatol. 2014;134(5):1332-1341.

37. Javelaud D, Laboureau J, Gabison E, Verrecchia F, Mauviel A. Disruption of basal JNK activity differentially affects key fibroblast functions important for wound healing. J Biol Chem. 2003;278(27):24624-24628.

38. Tachibana H, Perrino C, Takaoka H, Davis RJ, Naga Prasad SV, Rockman HA. JNK1 is required to preserve cardiac function in the early response to pressure overload. Biochem Biophys Res Commun. 2006;343(4):1060-1066.

39. Molkentin JD, et al. Fibroblast-specific genetic manipulation of $\mathrm{p} 38$ mitogen-activated protein kinase in vivo reveals its central regulatory role in fibrosis. Circulation. 2017;136(6):549-561.

40. Fink M, et al. A neuronal two P domain $\mathrm{K}+$ channel stimulated by arachidonic acid and polyunsaturated fatty acids. $E M B O J .1998 ; 17(12): 3297-3308$.

41. Maingret $\mathrm{F}$, et al. TREK- 1 is a heat-activated background $\mathrm{K}(+)$ channel. EMBO J. 2000;19(11):2483-2491.

42. Maingret F, Patel AJ, Lesage F, Lazdunski M, Honoré E. Mechano- or acid stimulation, two interactive modes of activation of the TREK-1 potassium channel. J Biol Chem. 1999;274(38):26691-26696.

43. Garry A, et al. Altered acetylcholine, bradykinin and cutaneous pressure-induced vasodilation in mice lacking the TREK1 potassium channel: the endothelial link. EMBO Rep. 2007;8(4):354-359.

44. Unudurthi SD, et al. Two-pore K+ channel TREK-1 regulates sinoatrial node membrane excitability. JAm Heart Assoc. 2016;5(4):e002865.

45. Rajabi M, Kassiotis C, Razeghi P, Taegtmeyer H. Return to the fetal gene program protects the stressed heart: a strong hypothesis. Heart Fail Rev. 2007;12(3-4):331-343.
46. Molkentin JD, et al. A calcineurin-dependent transcriptional pathway for cardiac hypertrophy. Cell. 1998;93(2):215-228.

47. Ling $\mathrm{H}$, et al. Requirement for $\mathrm{Ca} 2+/$ calmodulindependent kinase II in the transition from pressure overload-induced cardiac hypertrophy to heart failure in mice. JClin Invest. 2009;119(5):1230-1240.

48. Lorell BH, Carabello BA. Left ventricular hypertrophy: pathogenesis, detection, and prognosis. Circulation. 2000;102(4):470-479.

49. Weber KT, Janicki JS, Shroff SG, Pick R, Chen RM, Bashey RI. Collagen remodeling of the pressure-overloaded, hypertrophied nonhuman primate myocardium. Circ Res. 1988;62(4):757-765.

50. Díez J, Querejeta R, López B, González A, Larman M, Martínez Ubago JL. Losartan-dependent regression of myocardial fibrosis is associated with reduction of left ventricular chamber stiffness in hypertensive patients. Circulation. 2002;105(21):2512-2517.

51. Massie BM, et al. Irbesartan in patients with heart failure and preserved ejection fraction. $N$ Engl J Med. 2008;359(23):2456-2467.

52. Yin Z, Frasch M. Regulation and function of tinman during dorsal mesoderm induction and heart specification in Drosophila. Dev Genet. 1998;22(3):187-200.

53. Brand AH, Perrimon N. Targeted gene expression as a means of altering cell fates and generating dominant phenotypes. Development. 1993;118(2):401-415.

54. Wolf MJ, Amrein H, Izatt JA, Choma MA, Reedy MC, Rockman HA. Drosophila as a model for the identification of genes causing adult human heart disease. Proc Natl Acad Sci U S A. 2006;103(5):1394-1399.

55. Namiranian K, et al. Cerebrovascular responses in mice deficient in the potassium channel, TREK-1. Am J Physiol Regul Integr Comp Physiol. 2010;299(2):R461-R469.

56. Yoo B, et al. Beta1-adrenergic receptors stimulate cardiac contractility and CaMKII activation in vivo and enhance cardiac dysfunction following myocardial infarction. Am J Physiol Heart Circ Physiol. 2009;297(4):H1377-H1386.

57. Davis J, Burr AR, Davis GF, Birnbaumer L, Molkentin JD. A TRPC6-dependent pathway for myofibroblast transdifferentiation and wound healing in vivo. Dev Cell. 2012;23(4):705-715.

58. Golden HB, et al. Isolation of cardiac myocytes and fibroblasts from neonatal rat pups. Methods Mol Biol. 2012;843:205-214.

59. Rakesh K, Yoo B, Kim IM, Salazar N, Kim KS, Rockman HA. beta-Arrestin-biased agonism of the angiotensin receptor induced by mechanical stress. Sci Signal. 2010;3(125):ra46.

60. Acharya A, Baek ST, Banfi S, Eskiocak B, Tallquist MD. Efficient inducible Cre-mediated recombination in Tcf 21 cell lineages in the heart and kidney. Genesis. 2011;49(11):870-877.

61. Kraus WE, et al. A Guide for a cardiovascular genomics biorepository: the CATHGEN experience. J Cardiovasc Transl Res. 2015;8(8):449-457.

62. Kraus WE, et al. Metabolomic quantitative trait loci (mQTL) mapping implicates the ubiquitin proteasome system in cardiovascular disease pathogenesis. PLoS Genet. 2015;11(11):e1005553. 